\title{
A critical review on special forming processes and associated research for lightweight components based on sheet and tube materials
}

\author{
Lang Lihui, Liu Kangning*, Gaoshen Cai, Xiying Yang, Chan Guo, and Guoliang Bu \\ School of Mechanical Engineering and Automation, Beihang University, Beijing 100191, China
}

Received 4 May 2014 / Accepted 1 July 2014

\begin{abstract}
Lightweight materials, such as ultra-high strength steel, titanium alloys and aluminum alloys, are used widely in aerospace and automobile industries leading to increasing demands for advanced forming technologies. Recently many innovative sheet- and tube-forming methods have been proposed by researchers and R\&D facilities around the word. These methods can greatly improve the formability of materials with low plasticity and can produce complex-shaped parts with good surface qualities. This review aims at providing an insight into the latest developments of particular sheet- or tubular-metal forming process and shows basic applications in modern industry.
\end{abstract}

Key words: Review, Lightweight material, Sheet forming, Tube forming

\section{Introduction}

A current widespread trend is to use lightweight materials with the advantage of producing lightweight components to achieve the goal of reduction of emissions and energy. However, the poor formability of advanced lightweight materials at room temperature has seriously hampered their wider application. Lightweight materials, such as ultra-high-strength steel [1], titanium alloys [2, 3] and aluminum alloys [4] are used extensively in aerospace and automobile industries leading to increasing demands for advanced forming technologies, as conventional forming methods lack the ability to meet the need for fabricating lightweight structures. In the 21 st century, in metal forming area, many researchers and business R\&D facilities have proposed many advanced innovative forming methods dealing with this problem. These particular forming methods have both strengths and weaknesses in their suitability for different types of sheet metal forming processes.

This paper first categories three kinds of lightweight materials and then gives a detailed introduction to some of the particular sheet forming methods, including hydroforming, viscous pressure forming, solid granular-medium forming, superplastic forming, impact hydroforming and asymmetric incremental sheet forming. The state-of-the-art and current applications of

*e-mail: 1kn_buaa@163.com these processes are also discussed in addition to some current developments on the forming mechanisms.

\section{Lightweight materials}

\subsection{Aluminum alloys}

Aluminum alloys are effective materials for the reduction of vehicle weight and are expanding their applications over years (see Figure 1). Naka et al. [6, 7] investigated the effects of forming speed and temperature on the experimental forming limit diagram (FLD) for a fine grain Al-Mg alloy sheet by performing stretch-forming tests at various forming speeds at several temperatures. $\mathrm{Li}$ et al. $[8,9]$ investigated the uniaxial tensile deformation behavior of three aluminum sheet alloys including $\mathrm{Al}$ 5182, $\mathrm{Al} 5754$ and $\mathrm{Al}$ 6111-T4 in the warm-forming temperature range using the torsion test method, Cavaliere [10] studied the hot and warm formability of 2618 aluminum alloy, precipitation being found during deformation.

\subsection{Titanium alloys}

Titanium alloys possess the beneficial properties of high specific strength and excellent corrosion resistance. Therefore, titanium alloys are found in aerospace applications where the 


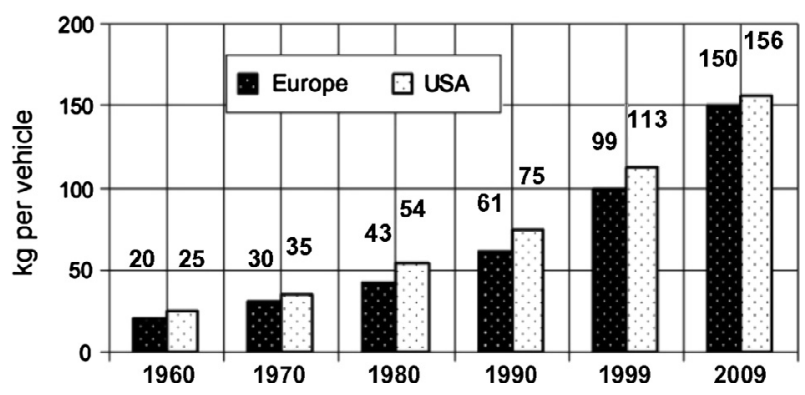

Figure 1. Average use of aluminum [5].
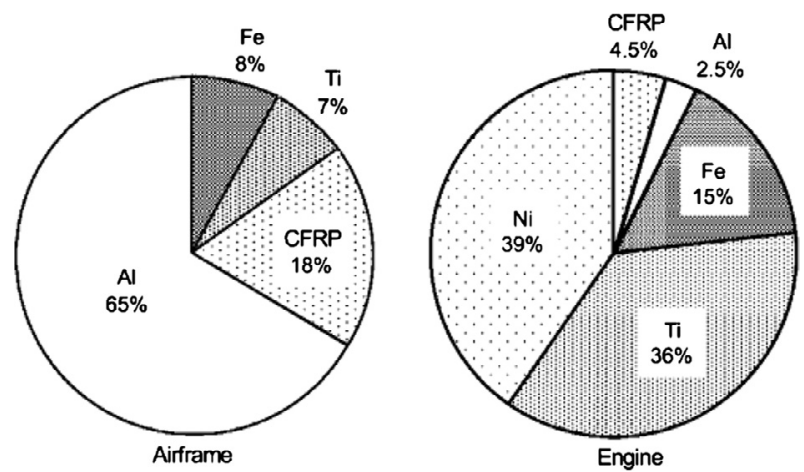

Figure 2. Percentage of aluminum, titanium, and steel alloys and carbon fiber reinforce plastic (CFRP) of the structural weight of modern large commercial aircraft and gas turbine engines [12].

combination of weight, strength, corrosion resistance and high temperature stability is highly valued [11].

Figure 2 shows the percentage of structural weight for various material classes in modern large commercial aircraft, distinguished between airframe and engine materials. The fuselage of the Airbus A330/340, for example, is manufactured of nearly two thirds aluminum. At about $7 \%$, titanium alloys have a similar share of the structural weight to that of steels. However, at over a third of the structural weight, titanium is the second most common material in the jet engine, following Ni-based superalloys; and by volume, titanium alloys are the most abundant material in the engine [12].

\subsection{High-strength steels}

In order to reduce vehicle weight and improve security, the increasing use of ultra-high-strength steels such as $22 \mathrm{MnB} 5$, $27 \mathrm{MnCrB} 5$, and $37 \mathrm{MnB} 4$ in the automotive industry has become an inevitable trend [13], Within this trend, advanced high-strength steel (AHSS) alloys have seen particularly fast growth [14], being used mainly in chassis components, such as the A-pillar, B-pillar, bumper, roof rail, rocker rail and tunnel (see Figure 3). Such prominent AHSS materials include dual phase, martensitic and boron steels [15]. Individual vehicle models and some companies have incorporated these advanced steels much more quickly than the fleet average: the Mercedes C-class jumped from $38 \%$ to $74 \%$ HSS in its body redesign,

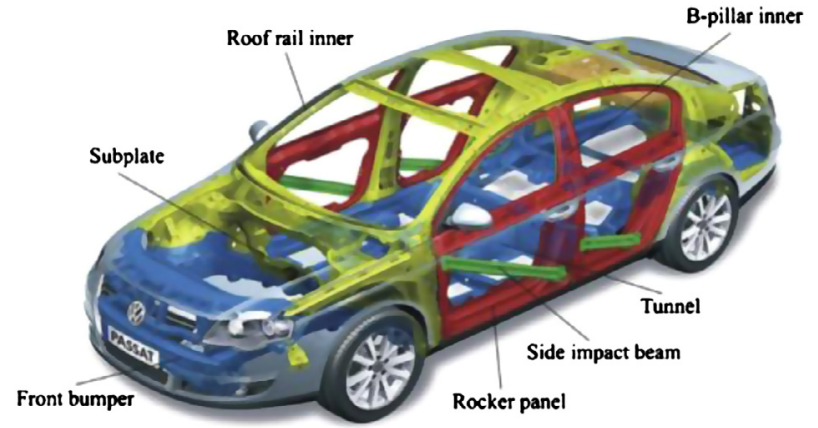

Figure 3. High strength steel used in a typical middle class car [13].

and the BMW X6 has $32 \%$ of its body and closure structures composed of AHSS [16]. Estimates from Ducker Worldwide indicate that the automobile industry will see an annual increase in AHSS of about $10 \%$ through to 2020 [17].

\section{Particular forming methods}

\subsection{Hydroforming process (HF)}

Hydroforming is a well-known long established process which was employed first to form sheets by the use of fluid medium as a soft punch before World War II. In recent years, with the reduction of cycle time and improvement of process controls, the fields of application of hydroforming have become broader. Hydroforming technology has gained increased interest in the world because of its many advantages.

Basically, the hydroforming process can be classified into two categories, sheet hydroforming and tube hydroforming [18], according to the different process features and the usage of different original blanks.

As shown in Figure 4a, a typical method in sheet hydroforming is hydraulic deep drawing (HDD). When the punch pushes the sheet metal into the die cavity, where oil or other liquids are contained, the established high pressure can press the sheet metal tightly onto the punch and a complex part will be formed. Another method is conventional sheet hydroforming (see Figure $4 \mathrm{~b}$ ), where, on the contrary, a rigid punch is replaced by pressurized liquids and the blank is bulged against a die.

Sheet hydroforming attracted widespread attention in the automobile industry when it was first applied to automotive parts as it provides products with relatively good surface quality compared with that of traditional deep drawing. Figure 5 [18] shows some automobile panels fabricated by the sheet hydroforming (SHF) process.

Recently, the enthusiasm generated by HDD technology has cooled down, mainly due to the reasons discussed below [18], miscalculations on these two aspects keeping the SHF process away from series production:

1. The cycle time of the SHF process is too long compared to that of conventional deep drawing or stretch drawing, restricting the SHF process to niche applications. 


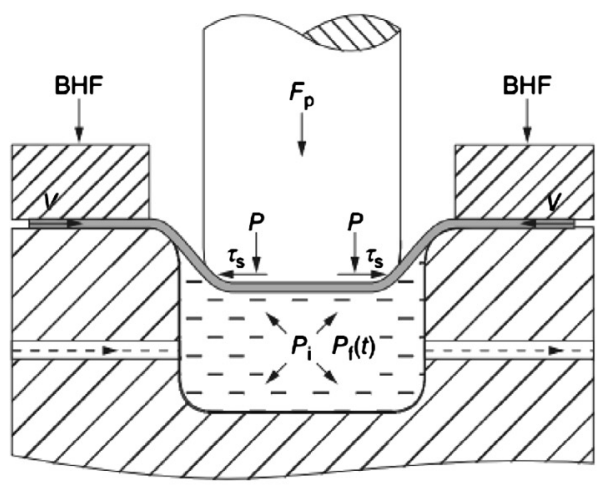

(a)

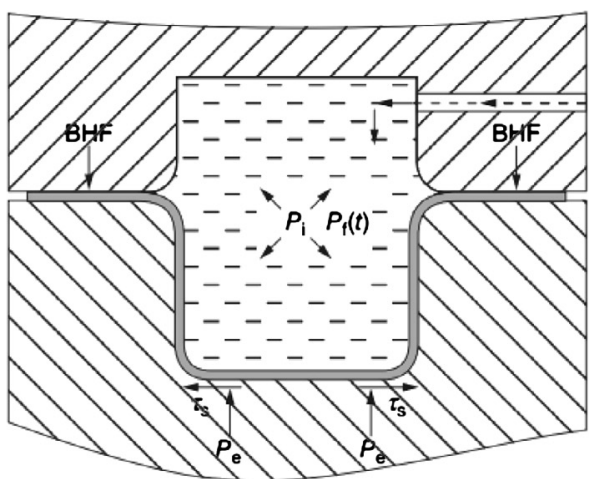

(b)

Figure 4. Sheet hydroforming process [19]. (a) hydraulic deep drawing, (b) conventional sheet hydroforming.

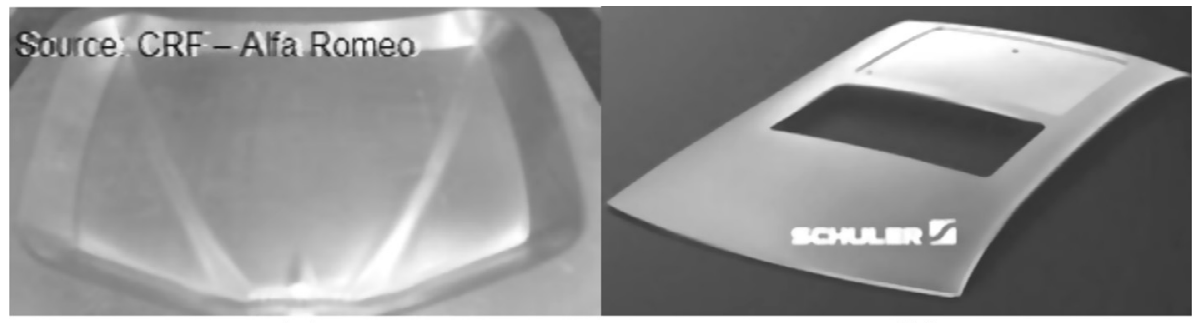

(a)

Figure 5. Application of sheet hydroforming (SHF) [18]. (a) Alfa Romeo hood, (b) Maybach roof.

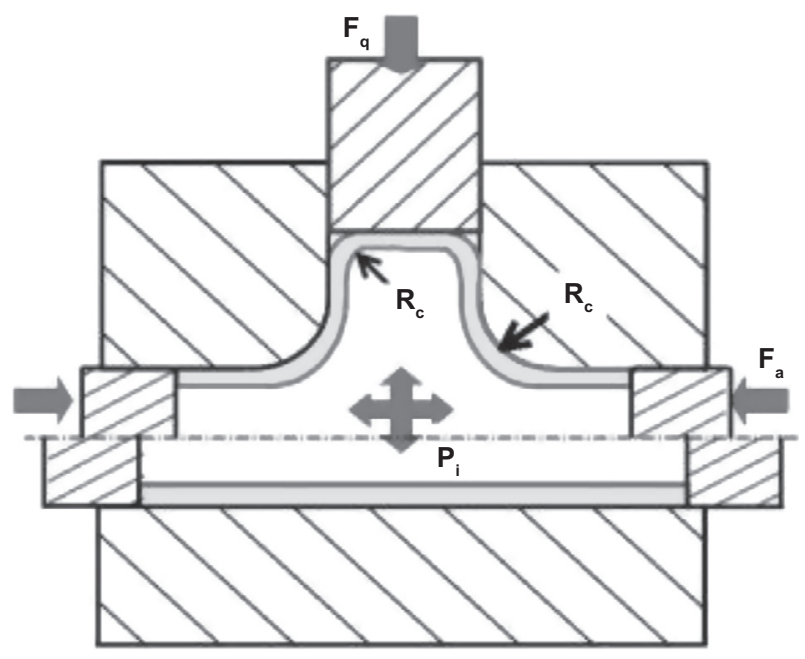

Figure 6. Typical THF process [20].

2. Special controlling parameters are needed in the process and these parameters have to be acquired by car manufacturers before application in commercialized production. However, the optimized data is not easy to obtain, this being the key point in making full use of the technique.

Figure 6 presents a schematic diagram of a typical tube hydroforming process. A seamless tube or a welded tube is used to form the shape of the die cavity by internal pressure with the corresponding movements of the side punches.

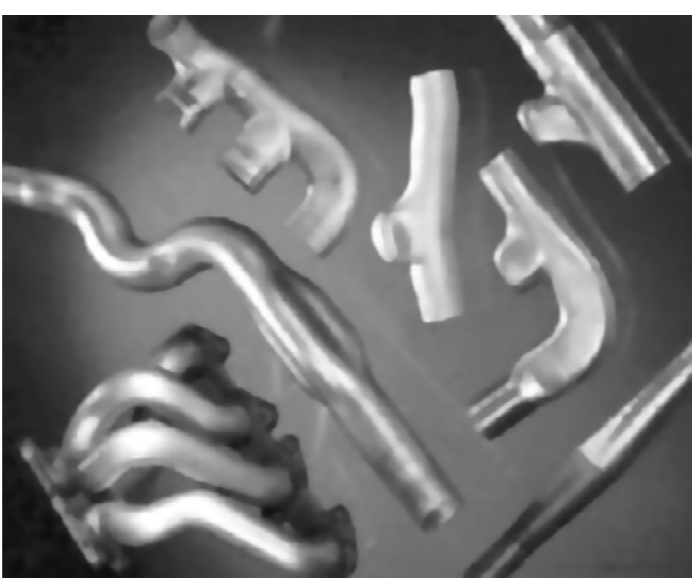

Figure 7. Exhaust parts produced by hydroforming (Schuler Hydroforming, Germany) [23].

Tube hydroforming has many advantages [21, 22] such as part consolidation, weight reduction, improved part strength. The process was first used in the automobile field for nonstructural components, such as exhaust and intake systems or cooling pipes (see Figure 7), since hydroforming is able to fabricate very complex part in a single operation, therefore reducing assembly cost and weight. Applying this process to structural components is a big challenge, but due to the R\&D investment of the automobile industry, many parts, such as suspension frames, A-pillars and engine cradles, have been 


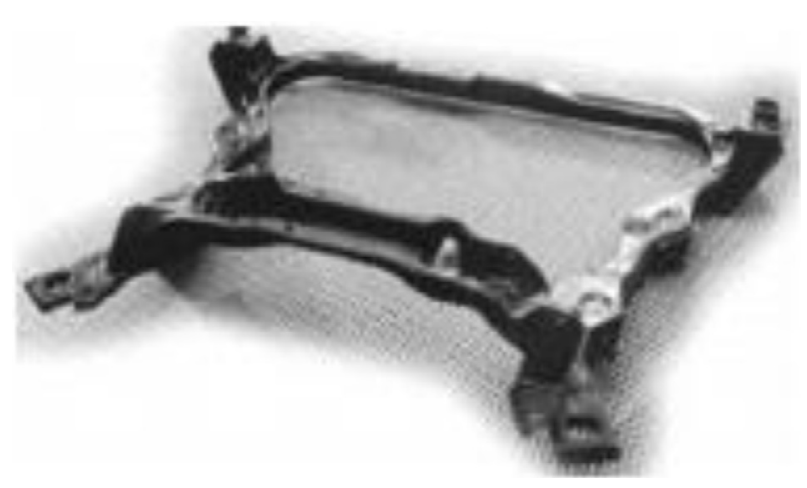

Figure 8. Engine cradle [23].

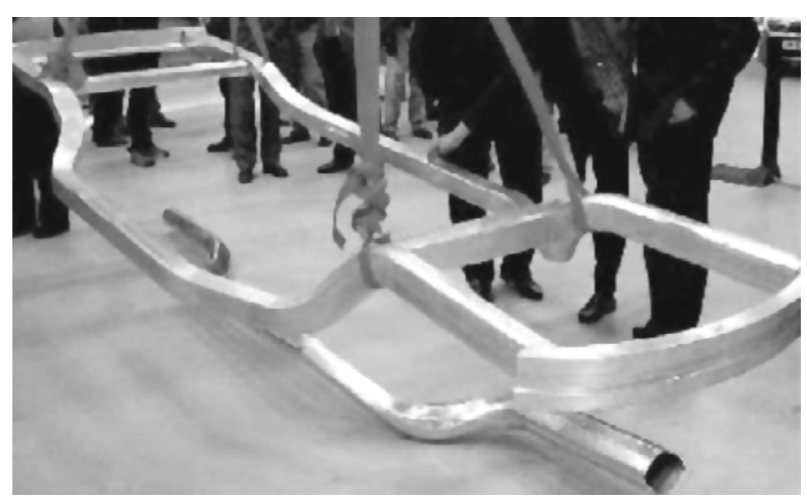

Figure 9. An aluminum lower rail for the Volvo car (AP\&T, Sweden) [23].

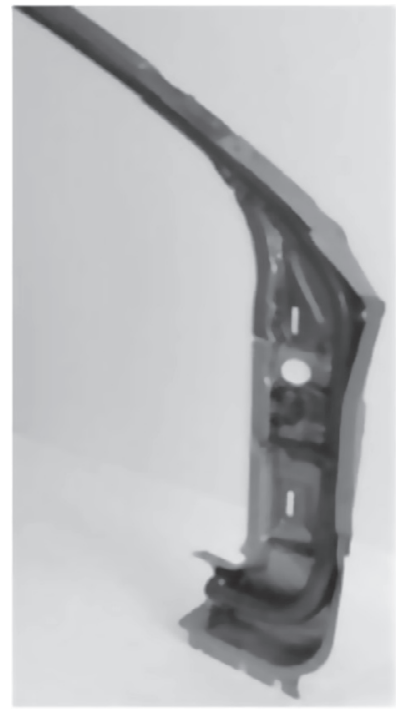

Conventional

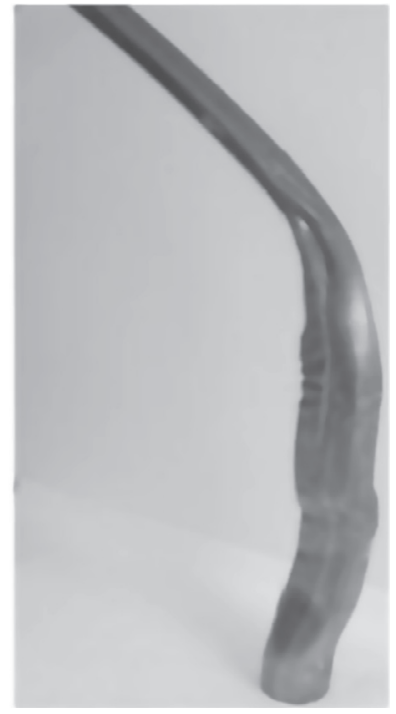

Hvdroformed
Figure 10. Comparison between a conventional A-pillar (left) and a hydroformed A-pillar (right) [18].

successfully developed (see Figures 8 and 9) which not only have the characteristic of being of light weight but also maintain the same structural performance.

Among these, one of the first research programs on this application was carried out in the early $2000 \mathrm{~s}$ as an EU-funded
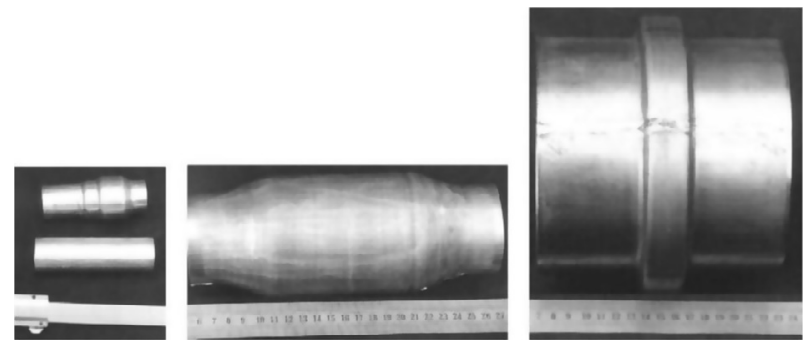

Figure 11. Hydroformed parts used in the aerospace industry (HIT, China) [23].

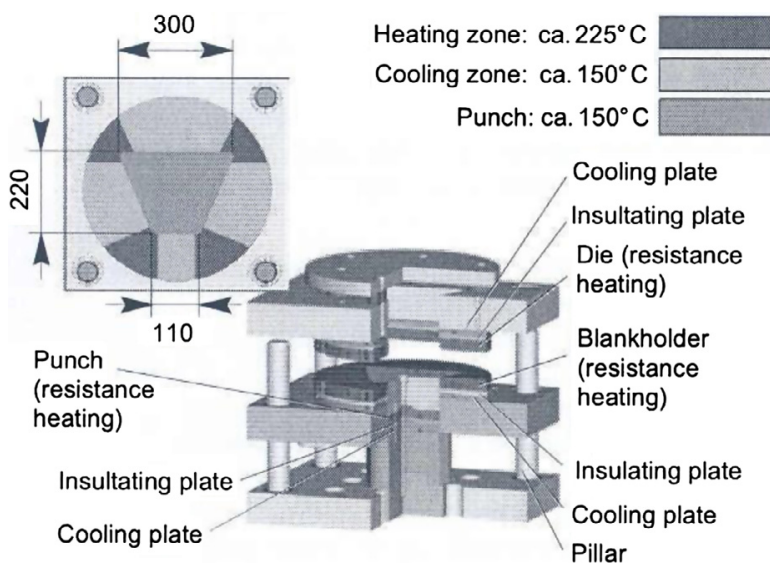

Figure 12. Deep drawing tool at elevated temperature [24].

(a)

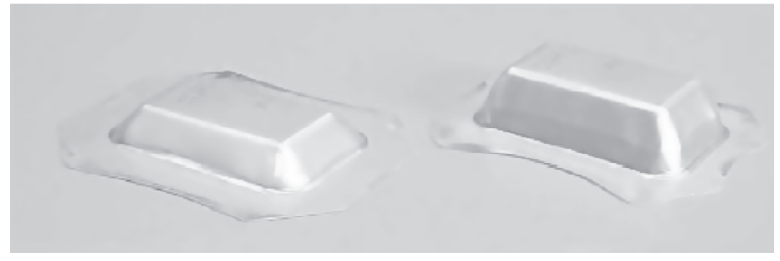

(b)

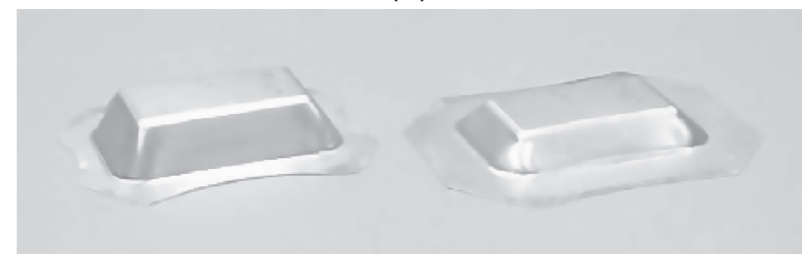

Figure 13. Formed aluminum boxes [25]. (a) Using 5754-O at room temperature (left) and $250{ }^{\circ} \mathrm{C}$ (right). (b) Using 6016-T4 at room temperature (left) and $175^{\circ} \mathrm{C}$ (right).

project and successfully produced the prototype of an A-pillar reinforcement realized by bending and hydroforming a conical tube (see Figure 10).

In addition to the automotive industry, hydroforming has also gained growing interest in the aerospace industry in the 


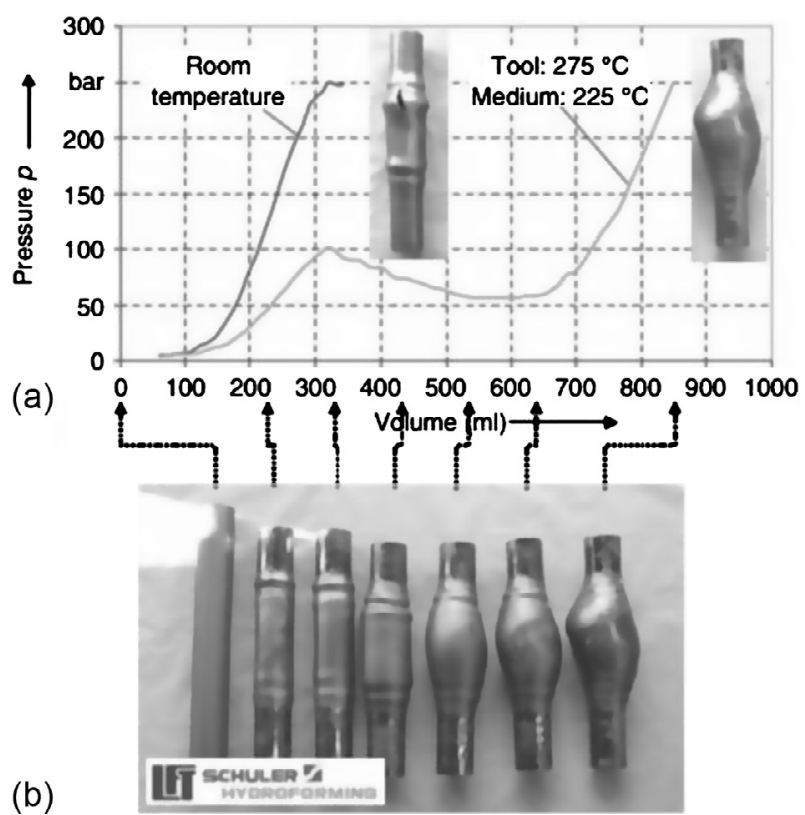

Figure 14. tube warm hydroforming test at room temperature and $220{ }^{\circ} \mathrm{C}[24]$. production of some complicated structural parts. The group led by Wang and Yuan in HIT (Harbin Institute of Technology) [23] has done many relevant researches on the application of hydroforming aircraft parts (see Figure 11), and many parts have been formed out of aluminum alloys or welded stainless tube blanks.

Another new trend is to combine warm forming with sheet/ tube hydroforming: the resultant process is called warm hydroforming, and can improve the formability of materials significantly. The tooling for conventional sheet metal forming at elevated temperature has been studied and developed for many years, all of which tooling is almost the same as that shown in Figure 12 [24].

Using 1050, 5754 and 6016 series aluminum sheets, Bolt et al. [25] investigated the formability at elevated temperatures of from 100 to $250{ }^{\circ} \mathrm{C}$. Both box and conical rectangular products were made with a tool with a heated die and blank holder. It was shown that forming at elevated temperature can yield a significant increase in product height, especially for conical products. The minimum die temperature for a significant effect on the process limit is lower for the Al6016 alloy than for the Al5751 alloy. The hardness of the warm-formed products does not differ significantly from that of products shaped at room
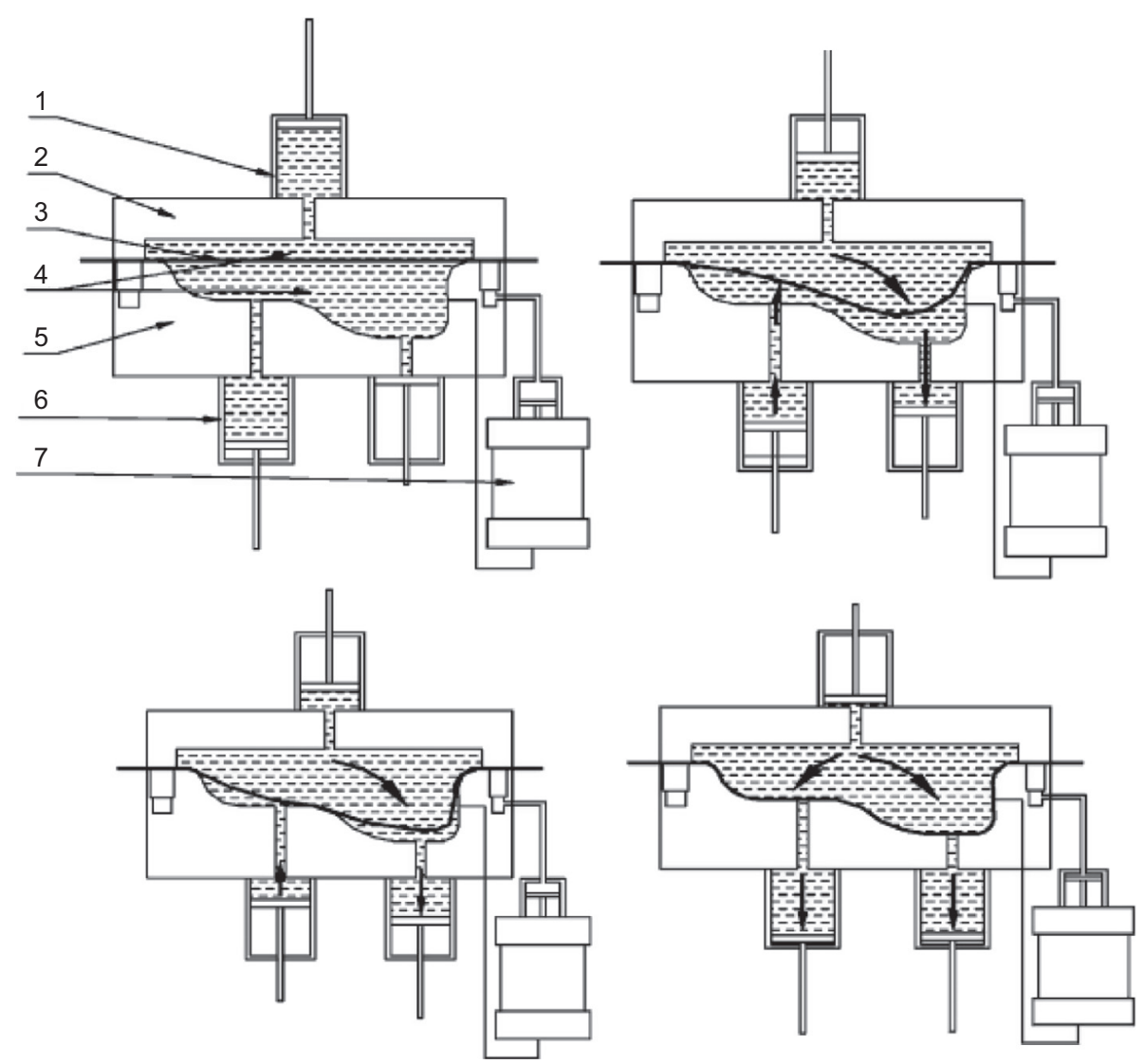

Figure 15. A schematic diagram of VPF [26]: (1) medium injection cylinder; (2) upper die; (3) blank sheet; (4) viscous medium; (5) die; (6) medium outlet cylinder; (7) blank holder cylinder. 
(a)

(b)

(c)

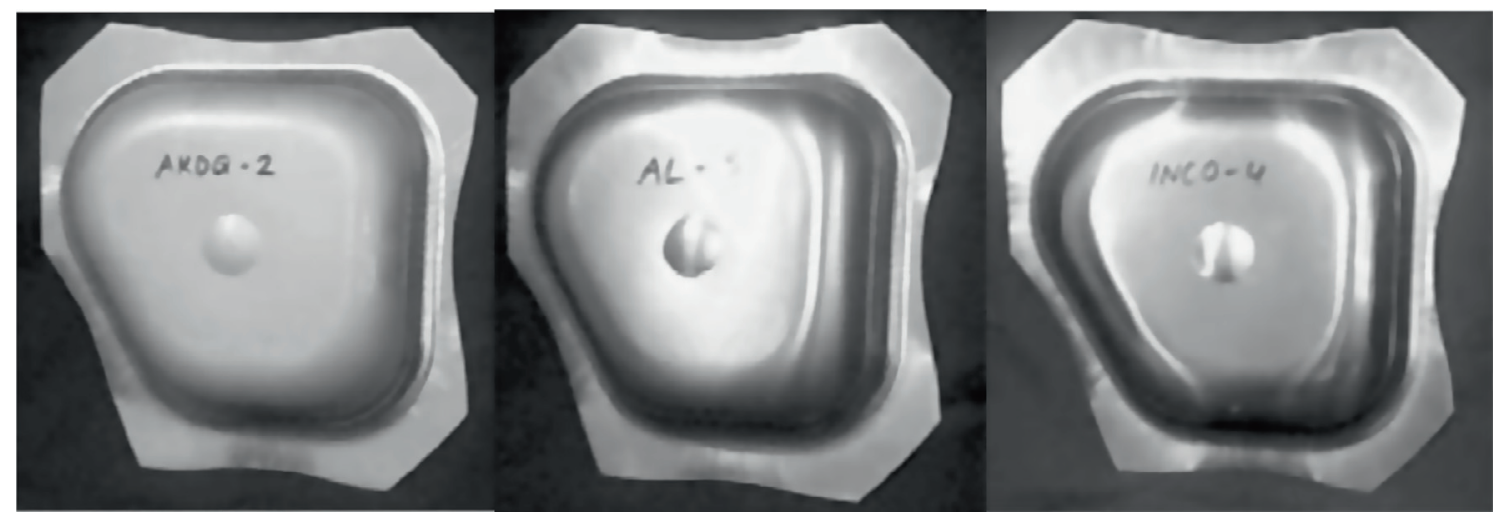

Figure 16. SPF parts formed in VPF tooling [30]. (a) AKDQ steel, (b) Aluminum alloy 6111-T4, (c) Inco 718.

(a)

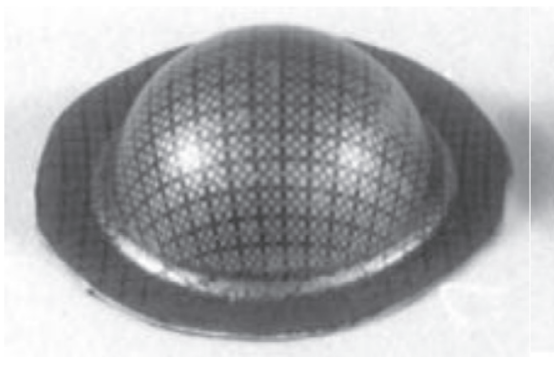

(b)

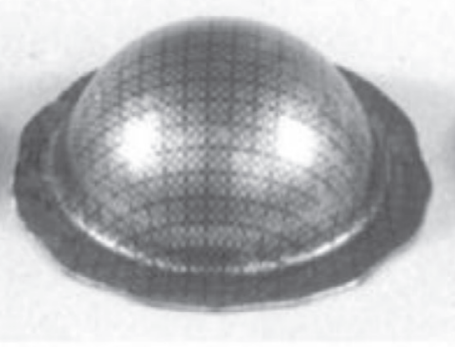

(c)

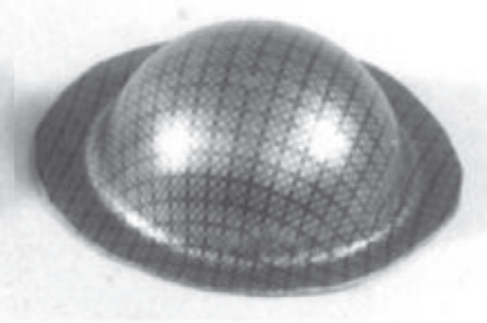

Figure 17. Parts obtained in the bulging experiment [32]. (a) Viscous medium bulging part, (b) polyurethane bulging part, (c) steel bulging part.

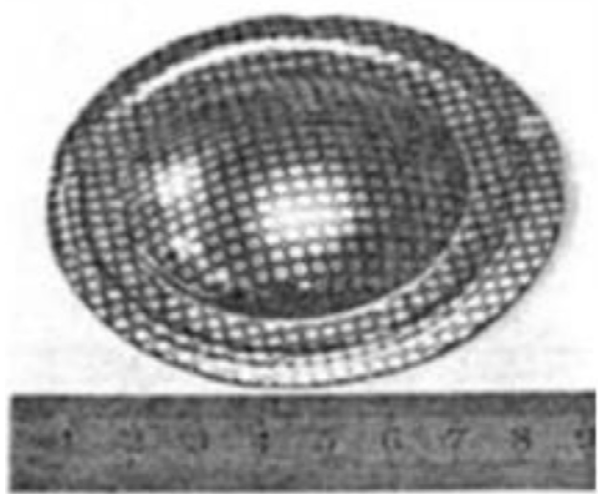

Figure 18. VPF specimens at elevated temperature [33].

temperature. Figure 13 shows the deepest-formed conical boxes using Al5754 and Al6016.

Kleiner et al. [24] conducted bulging tests of tube hydroforming at elevated temperatures. The tube material was an $\mathrm{Al}$ alloy (AlMg3.5Mn) and was welded and annealed before the tests in order to secure good formability. Figure 14 shows the pressure curve versus the injected volume for warm tube hydroforming at room and elevated temperature. At room temperature, the pressure increased very quickly and failure of the tube occurred very soon in the process. By increasing the tool temperature to $275{ }^{\circ} \mathrm{C}$ and the temperature of the pressurized medium within the tube to $225^{\circ} \mathrm{C}$, the formability of the tube increased significantly.

\subsection{Viscous pressure forming (VPF)}

The VPF process was invented in 1992 in the form of a patent and it is differed from conventional deep-drawing processes by applying viscous materials as the force-transmitting medium. Specifically, in the VPF process, high pressure exerted by viscous medium is applied on one side of a sheet metal blank to replace rigid punches, whilst back-pressure is also exerted to improve formabilities (see Figure 15). The viscous-medium forming method can make complex-shaped components with good surface quality and high dimensional accuracy, and at the same time reduce leakage [27]. Furthermore, the VPF process also has the advantage of producing parts made of high strength and in other light metals which are difficult to form by conventional sheet metal forming methods $[28,29]$.

Ahmetoglu et al. [30] conducted VPF experiments on a non-symmetrical part at the ERC/NSM laboratory using three different materials, namely AKDQ steel, aluminum alloy 6111-T4 and Inco 718 SPF and studied the influence of process parameters, such as clamping load, forming speed and sheet 


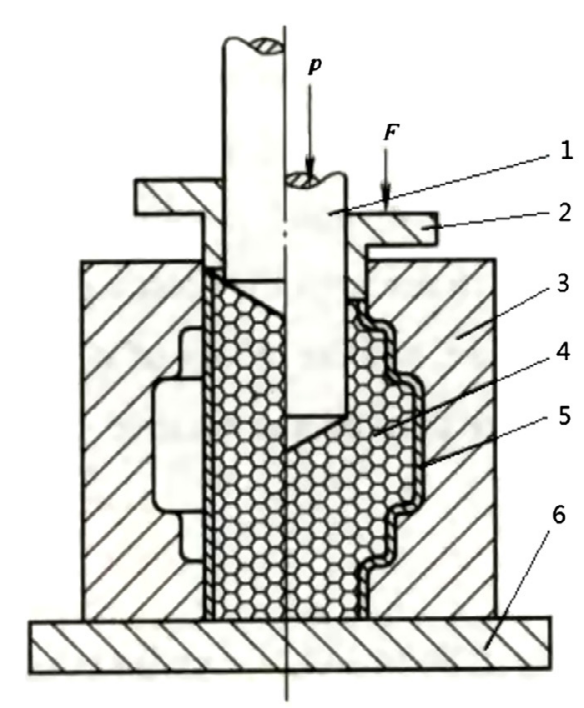

Figure 19. Photo of deformed typical parts [34].

(a)

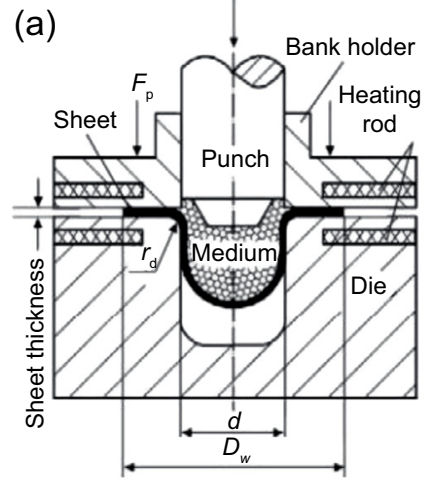

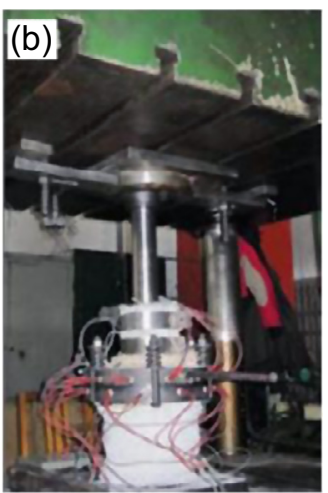

Figure 20. Schematic diagram of deep drawing and experimental equipment of AZ31B magnesium alloy [36]: (a) Diagram of deep drawing, (b) experimental equipment of deep drawing.

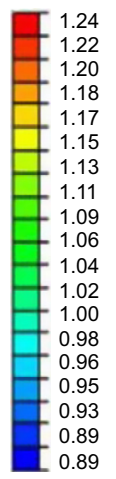

Figure 21. Deep drawing workpiece and contours band of thickness [36].

metal blank material, on the technique (see Figure 16). Shulkin et al. [31] introduced an eight-point BHF control system to an experimental VPF machine and studied the formability by both
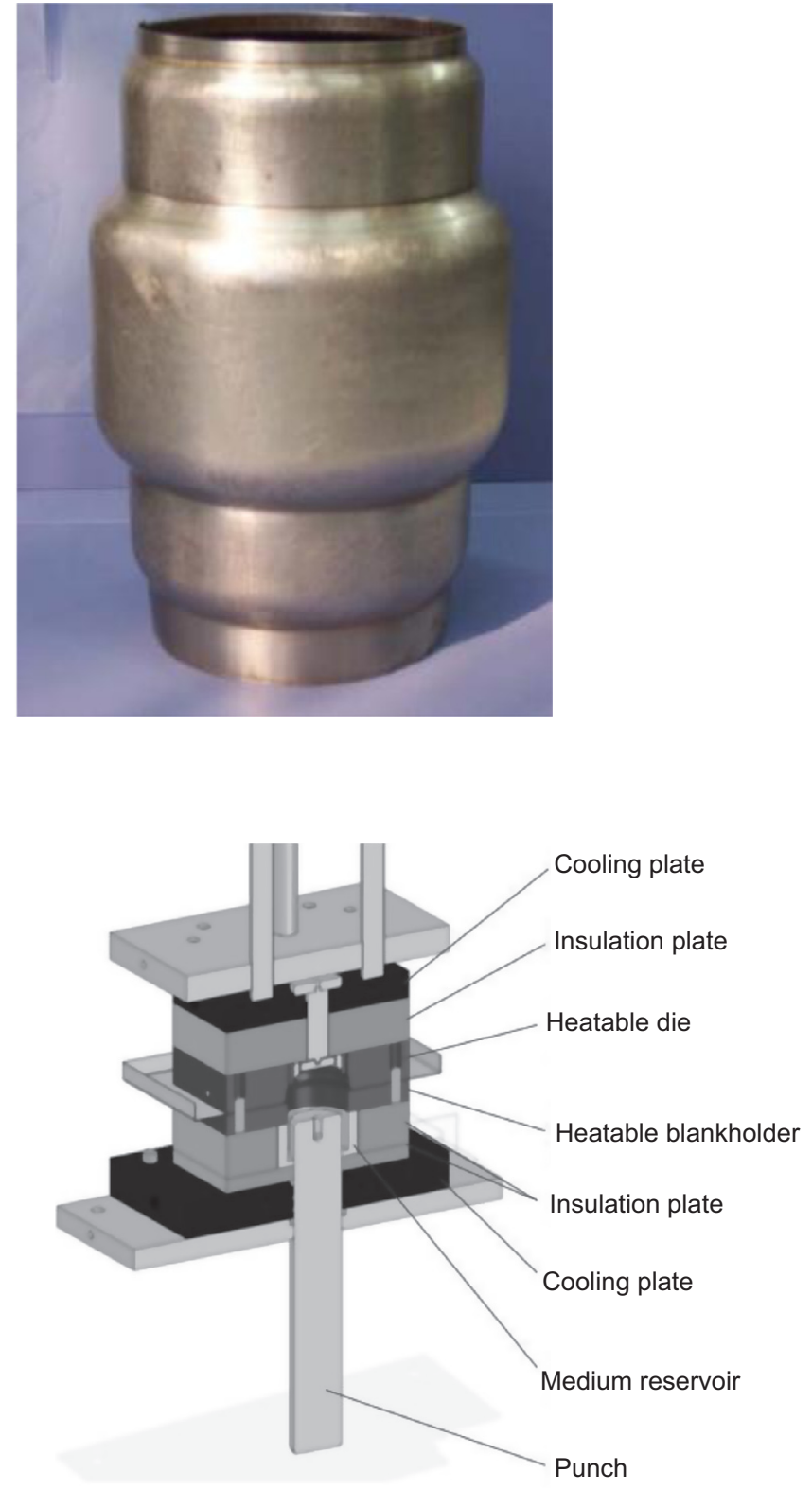

Figure 22. Experimental tool for hydroforming using granular material as a medium at elevated temperatures [37].

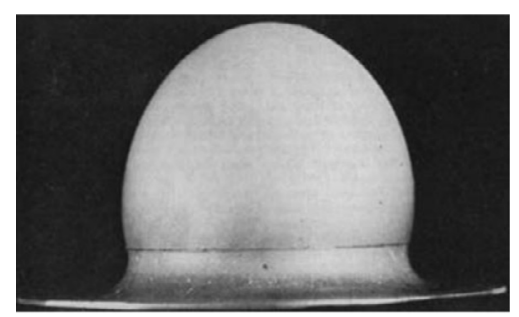

Figure 23. The first superplastically formed bubble [41].

simulation and experiment. Contrast experiments of bulging tests with three kinds of pressure-carrying media, viscous medium, polyurethane and steel, was studied by Wang et al. [32], 


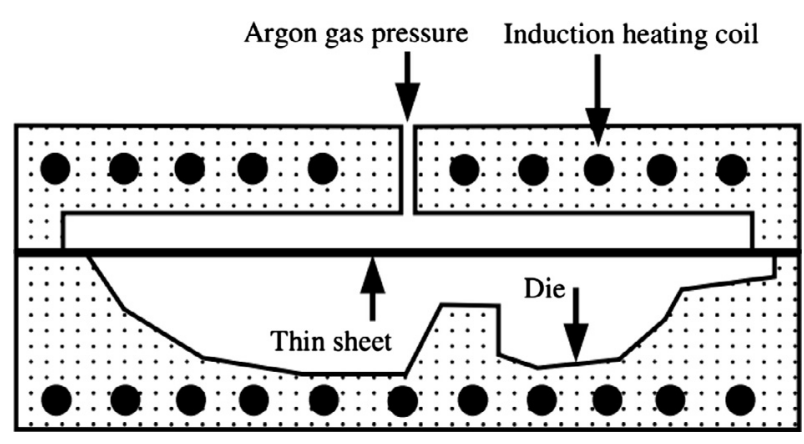

Figure 24. Schematic of SPF process [42].

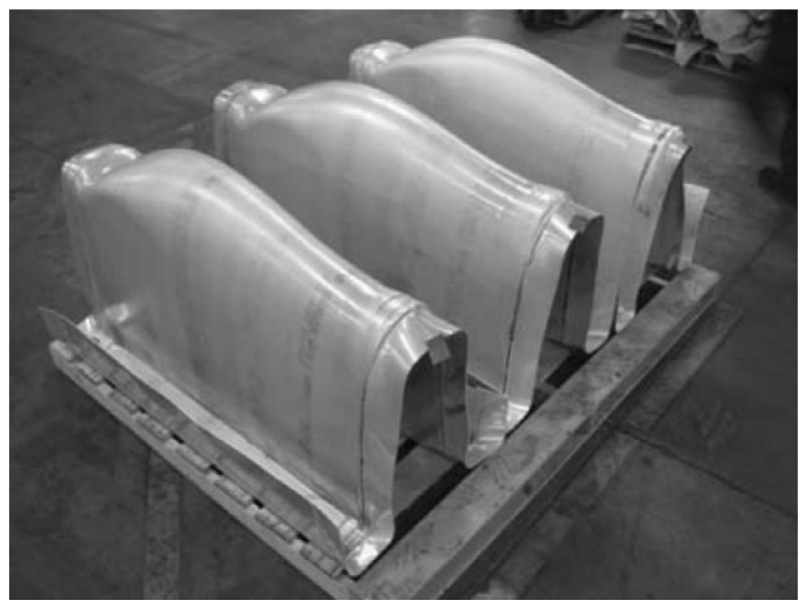

Figure 25. Non-planar clamp line SPF forming [43].

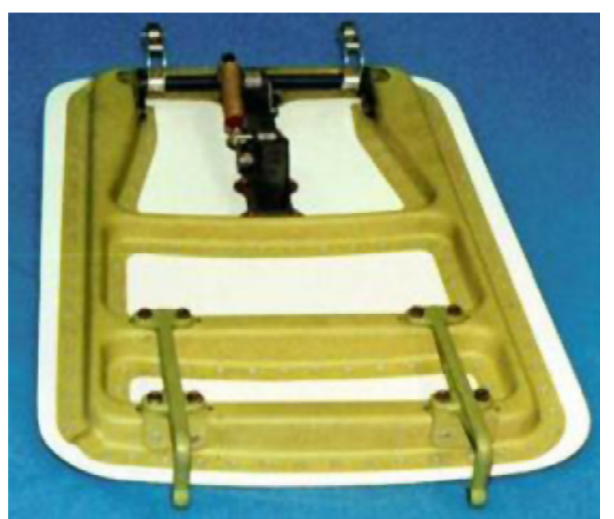

Figure 26. 737 blowout door [45].

the results showing that specimens formed with VPF have less wall-thinning and consequently have a more uniform wall thickness distribution compared with those formed with polyurethane and steel (see Figure 17).

Liu et al. [33] improved VPF technology by using the process at elevated temperature to form an aluminum alloy sheet metal (see Figure 18). The limiting dome height (LDH) of test specimens was measured to evaluate the formability of VPF at different temperatures. Results showed that the LDH of bulging specimen at $200{ }^{\circ} \mathrm{C}$ attained $17.4 \mathrm{~mm}, 10.3 \%$ higher than that at room temperature. The influence of forming temperature on thickness distribution, forming load and failure location of sheet metal was also investigated. To the present day, the VPF process has basically remained in the research stage and has not been used extensively in the aerospace or automotive industries.

\subsection{Solid granular medium forming (SGMF)}

The innovative technology of Solid Granular Medium Forming (SGMF) was proposed to overcome the disadvantages of leakage problems and poor heat resistibility of hydroforming by Zhao et al. [34-36]. In this process, a granular medium replaces water or oil or viscous materials as the forcetransmitting medium. Considering that fluids normally used at room temperature are only temperature stable up to about $350{ }^{\circ} \mathrm{C}$, using solid granular medium allow high temperatures and can totally reduce the risk of leakage. Similarly to the hydroforming method, this technology can fabricate both tubular parts (see Figure 19) and sheet parts (see Figure 20). Moreover, the non-uniform distribution of the internal pressure of solid granules can significantly improve the formability of the process, in addition, the interaction between the solid granular medium and the sheet metal surface can also greatly reduce the thinning of the workpiece [35].

By conducting SGMF tests at warm temperatures, Cao et al. studied the granular medium forming performance of magnesium alloy sheet (see Figure 21) and determined that AZ31B magnesium alloy sheet achieves the best deep drawing performance under the conditions of a forming temperature of from $250{ }^{\circ} \mathrm{C}$ to $300{ }^{\circ} \mathrm{C}$. These authors also found that the granular medium can apply axial friction on the cylinder wall to further improve the forming performance of metal sheet [36].

Merklein et al. [37, 38] used ceramic beads as a forming medium and designed and assembled an experimental apparatus (see Figure 22) to conduct the process at elevated temperatures. The apparatus can make SGMF parts at temperatures of up to $600{ }^{\circ} \mathrm{C}$, when the lightweight materials such as high- and ultra-high-strength steel or titanium are suitable for forming.

\subsection{Superplastic forming (SPF/DB)}

Superplasticity is a characteristic of some fine-grained (3-5 $\mu \mathrm{m})$ alloys when specimens under low stress conditions can exhibit neck-free elongations of very large values [39, 40]. Since the first superplastic part was made pneumatically from a sheet of superplastic Al-Zn eutectoid alloy 50 years ago [41] (see in Figure 23), this technology has been actively pursued in academic, research organizations and industrial R\&D facilities around the world due to its special advantages of meeting some unique requirements in the aerospace and automobile industry.

A schematic diagram of the SPF process can be seen in Figure 24, where a thin piece of sheet is put into the tools on a press and sealed at the edges, then the chamber is filled with high pressure gas at elevated temperature. The sheet is gradually deformed in a like manner to the blowing of bubble gum. The commercially available alloys for the SPF process 


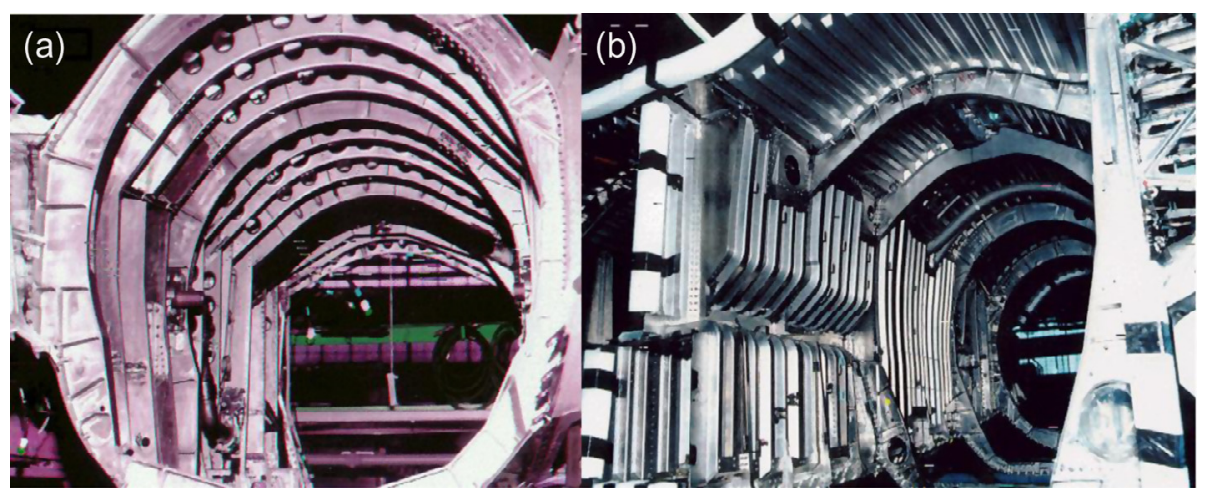

Figure 27. The use of superplastic forming-diffusion bonding to reduce the fuselage part count in F-15E aft fuselage (blats SPF-DB) showing (a-left) before and (b-right) after SPF-DF [46].

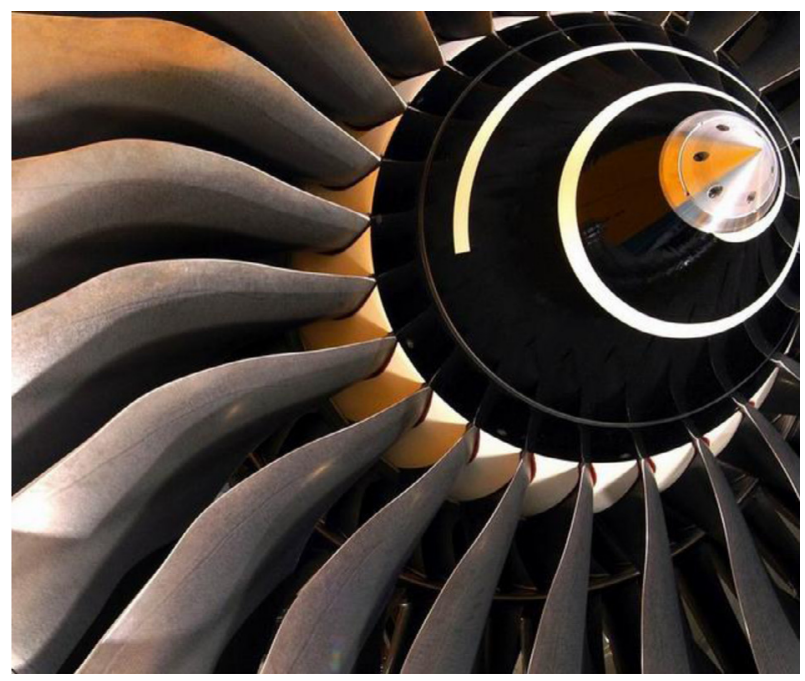

Figure 28. The titanium fan blade for Trent 900 engine.

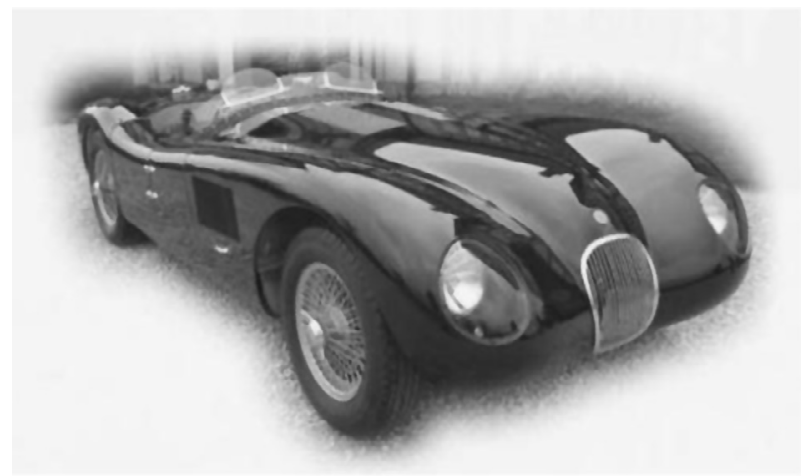

Figure 29. The 2006 limited series reproduction of the 1954 classic Jaguar C-Type [43].

are usually non-ferrous metals such as: aluminum alloy, titanium alloy and magnesium alloy [43].

In general, the SPF process can produce parts with complex geometry and thus replace multi-piece assemblies with one

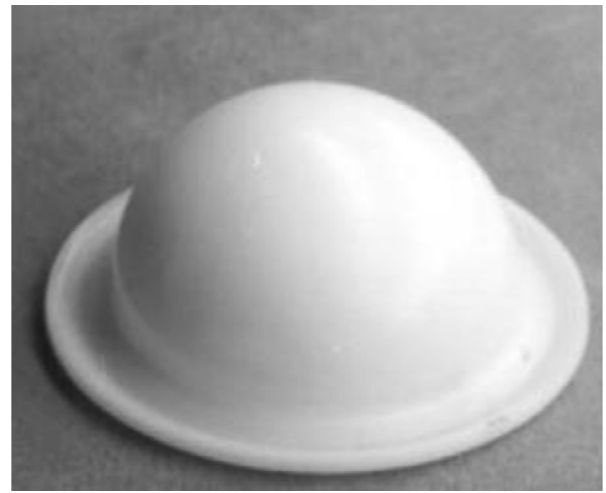

Figure 30. First bubbles superplastically formed from ceramic sheet.

monolithic component, without the need for welding or riveting parts together. Moreover, the components made by the SPF process can contain very little residual stress and have no springback [44]. The technology for SPF with aluminum alloys has progressed significantly in recent years. More often a nonplanar clamp line tooling arrangement comprising an upper and a lower tool is used to replace planar tools to manufacture complex parts with curving edges (see Figure 25).

Perhaps aircraft manufacturing companies may have the most interest in this technique. The first SPF aluminum component fabricated by Boeing for civil aircraft was in 1992 [45], shown in Figure 26. Up to now, the emphasis at Boeing has focused on developing applications for the 5083 alloy because of its non-heat-treatable property, and many parts such as the 737 wing-tip light housing, the 767 moisture shroud, etc. [44] are manufactured in the SPF process, to save both cost and weight. SPF technology is often used along with diffusion bonding (SPF/DB) to further enhance the forming ability. After applying the SPF/DB process in building the F-15E fighter, it is estimated that about 726 parts and 10,000 fasteners are eliminated (Figure 27). The SPF process also made the wide-chord hollow fan blade possible in aeroengines (see Figure 28). 
(a)
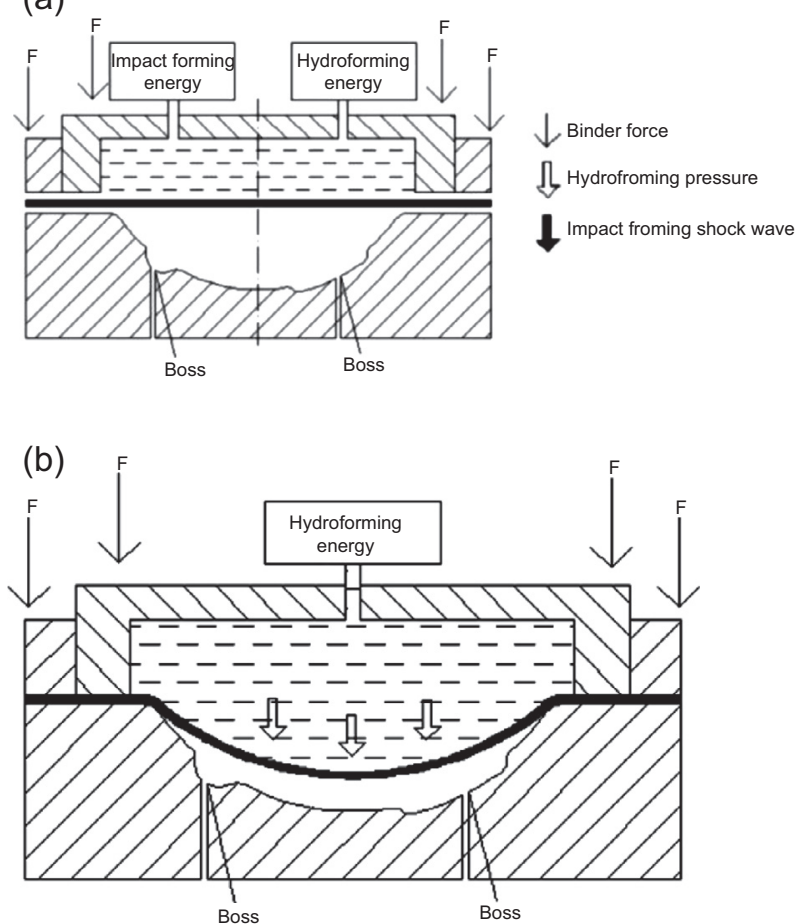

(c)

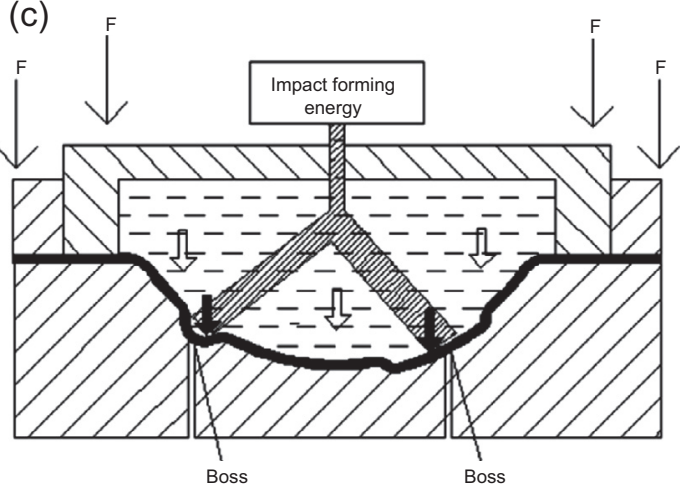

Figure 31. Steps of impact hydroforming technology [50]. (a) Preparation before forming. (b) The hydroforming step. (c) The impact forming step.

The promotion of the SPF process is limited in the automobile industry and only niche markets, including specialist vehicles and limited-series sports cars, are suitable for SPF applications [47]. Figure 29 shows the 2006 limited-series reproduction of the 1954 classic Jaguar C-Type in which the SPF process is applied [43]. The reasons why the SPF process is not suitable for Production cars are listed below:

1. The production rate is low and usually for low/medium volume applications.

2. Unique requirements for the materials, which are relatively expensive.

3. The advantage of forming complex part is not highly valued in high-volume models.

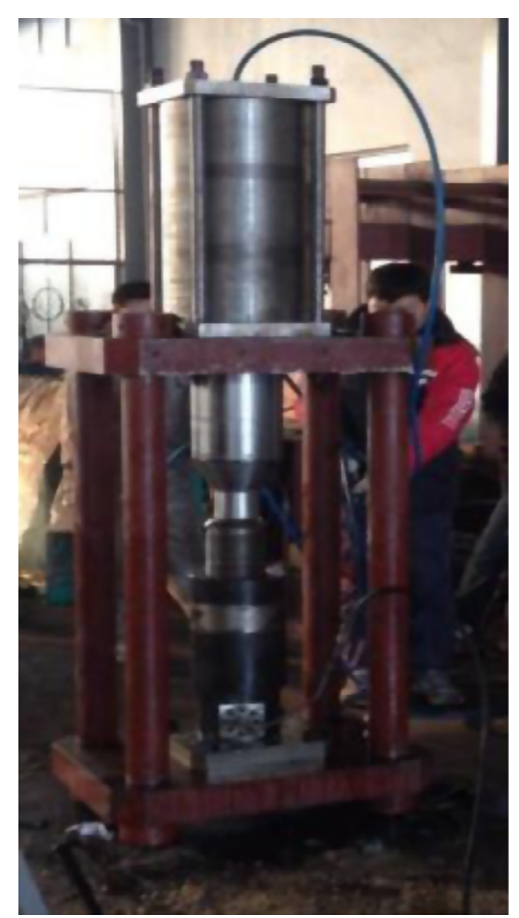

Figure 32. The IHF equipment.

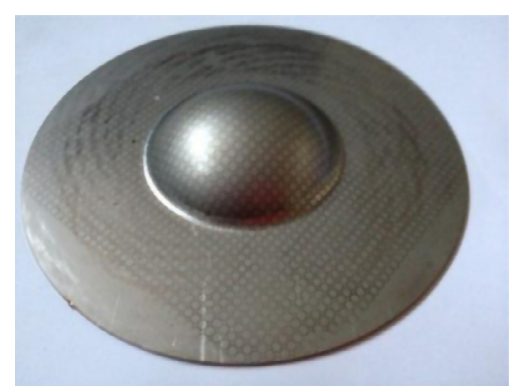

Figure 33. Formed part by IHF process.

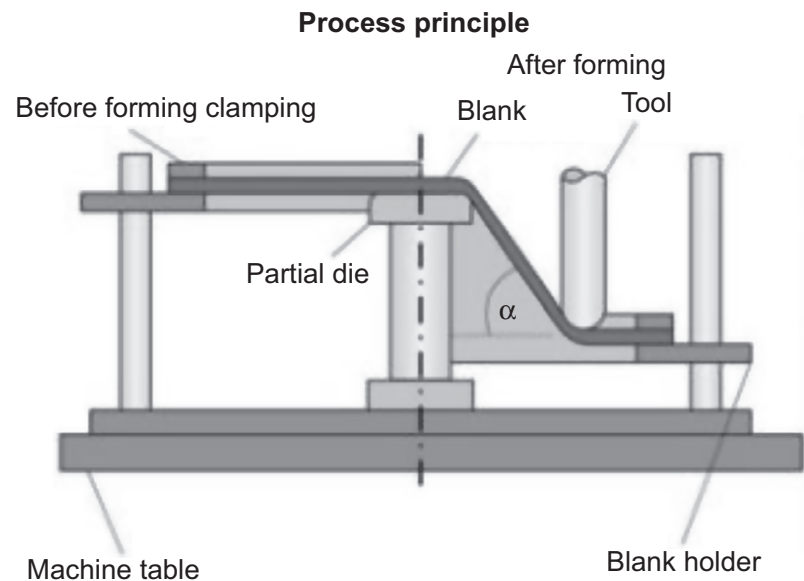

Figure 34. Process principle of AISF process [54]. 


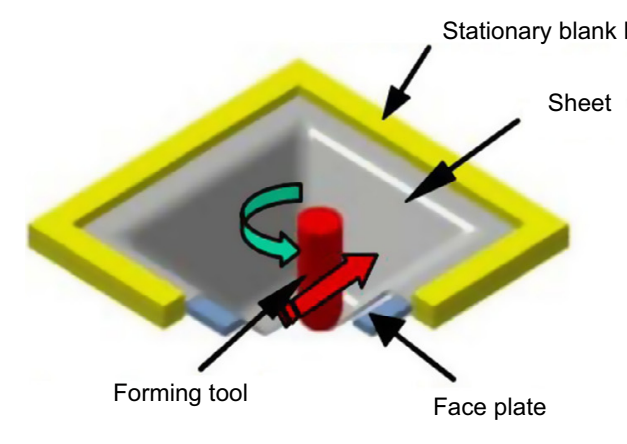

(a)

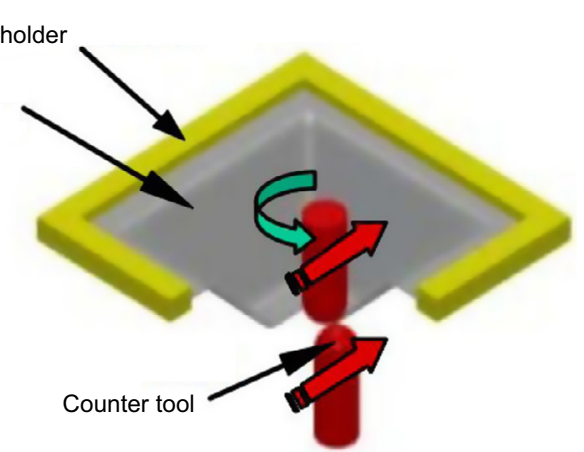

(b)

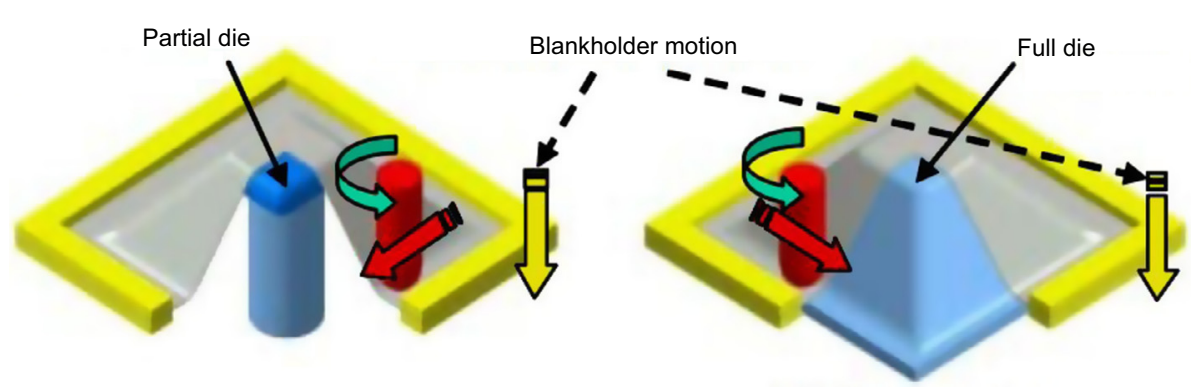

(c)

(d)

Figure 35. Four categories of AISF process [55]. (a) Single point incremental forming; (b) Incremental forming with counter tools; (c) Two point incremental forming (partial die); (d) Two point incremental forming (full die).

Today the materials used for the SPF process are not limited to metal alloys. Non-metal materials, such as superplastic ceramics [47] (see Figure 30) and glassy metals [49], also demonstrate their ability in the SPF process.

\subsection{Impact hydroforming method (IHF)}

The innovative method of impact hydroforming (IHF) was proposed by Lang et al. [50] to meet the requirements of forming complex parts with small features which are very common in the automotive and aircraft industries. In the process, most of the geometrical features are formed by hydroforming (see Figure $31 \mathrm{~b}$ ) and some small fillet radius features are rapidly reshaped by high intensity impact energy (see Figure 31c) in a very short time. They also designed an IHF test setup (see Figure 32) to conduct experiments into the process (see Figure 33).

The original idea of impulsive pressure forming can be traced back into the 1960s [50], thus the IPF process can be viewed as a developed method in combination with hydroforming. Moreover, compared with traditional hydroforming methods, impact hydroforming technology can greatly improve the forming speed and the forming accuracy of sheet-metal component. The loading is quite short - less than $10 \mathrm{~ms}$ - and strict sealing is not required for the equipment. Moreover, the uniformity of wall thickness of the components can also be assured, as liquid medium fills the whole die cavity and establishes welldistributed pressure [50].

\subsection{Asymmetric incremental sheet forming (AISF)}

The Asymmetric Incremental Sheet Forming (AISF) process was first described by Mason in 1978 [52] and was developed mainly for the needs of the low volume production of sheet-metal parts. In the AISF process, a metal sheet is placed in the tooling and clamped at the edges, then a tool moves following the required shape in space under $\mathrm{CNC}$ control by a succession of "planar" contours or a single "spiral" contour so that the part is obtained as the result of accumulated localized plastic deformations [53]. The AISF process (see Figure 34) is similar to the spinning process but, with the absence of workpiece rotation, asymmetric components can be manufactured by the independent controlling of the $X, Y$ and $Z$ axis movements. One significant advantage of this technology is the ability to form asymmetric shapes at low cost, without the need of expensive dies.

Basically, two main variations of conventional AISF are distinguished: "single point incremental forming" (SPIF) and "two point incremental forming" (TPIF) [54]. Figure 35 shows the different configurations that are included in the group of ASIF techniques. In the SPIF process, the blank edge is clamped without any further support except for the forming tool 

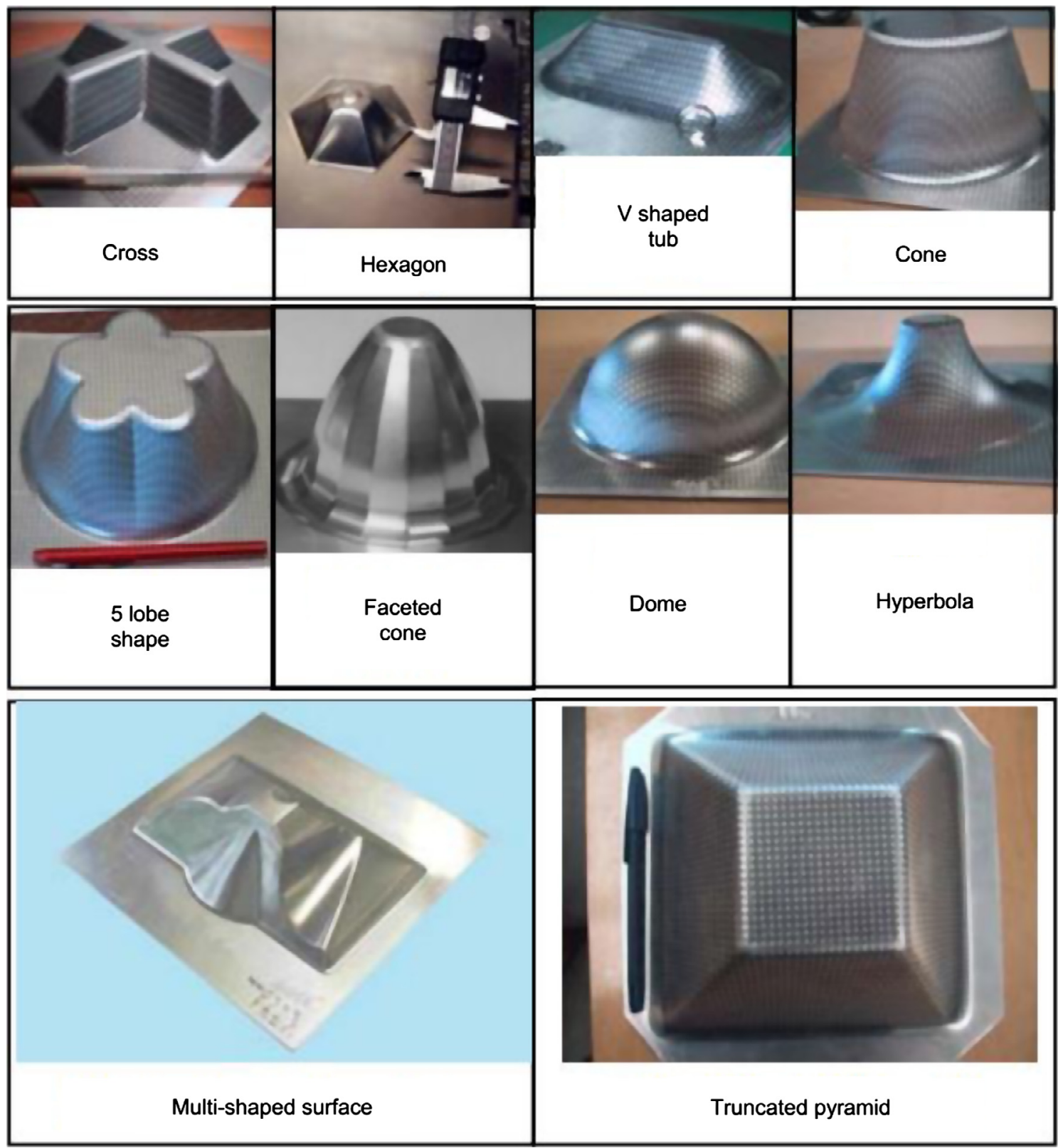

Figure 36. Shapes used to demonstrate the ability of the process and for experiments [60].

(see Figure 35a) [55]; whilst Figure 35b presents a special AISF process with counter tools. Up to now, considerable achievements of this process have been made by Kim and Yang [56], Leach et al. [57], and Felici et al. [58]. In the TPIF process (also called the backward bulge method by Matsubara [59]), there may be a simple support or a partial or full die (see Figures $35 \mathrm{c}$ and $35 \mathrm{~d}$ ). Both symmetric and asymmetric shapes can be fabricated using the TPIF or SPIF processes. The configuration shown in Figures 35c and 35d are usually associated with machines dedicated to the incremental forming process, and those in Figures $35 \mathrm{a}$ and $35 \mathrm{~b}$ are usually modified in a $\mathrm{CNC}$ milling machine [60].

As mentioned above, the major advantage of asymmetric incremental forming is the property of making asymmetric parts quickly and economically, without using expensive dies.
Shapes used to demonstrate the abilities of the process are shown in Figure 36 [60]. Now the AISF process has been used for designs that were made as Rapid Prototypes for the automotive industry [61] (see Figure 37) and the medical industry [62] (see Figure 38).

\section{Fundamental research for special sheet and tube forming}

In addition to the specific methods of implementing these special sheet and tube forming processes, emphasis has also been paid on the fundamental research concerning those techniques, such as the deformation mechanism, forming limit study, macro-deformation and micro-structure evolution of the 


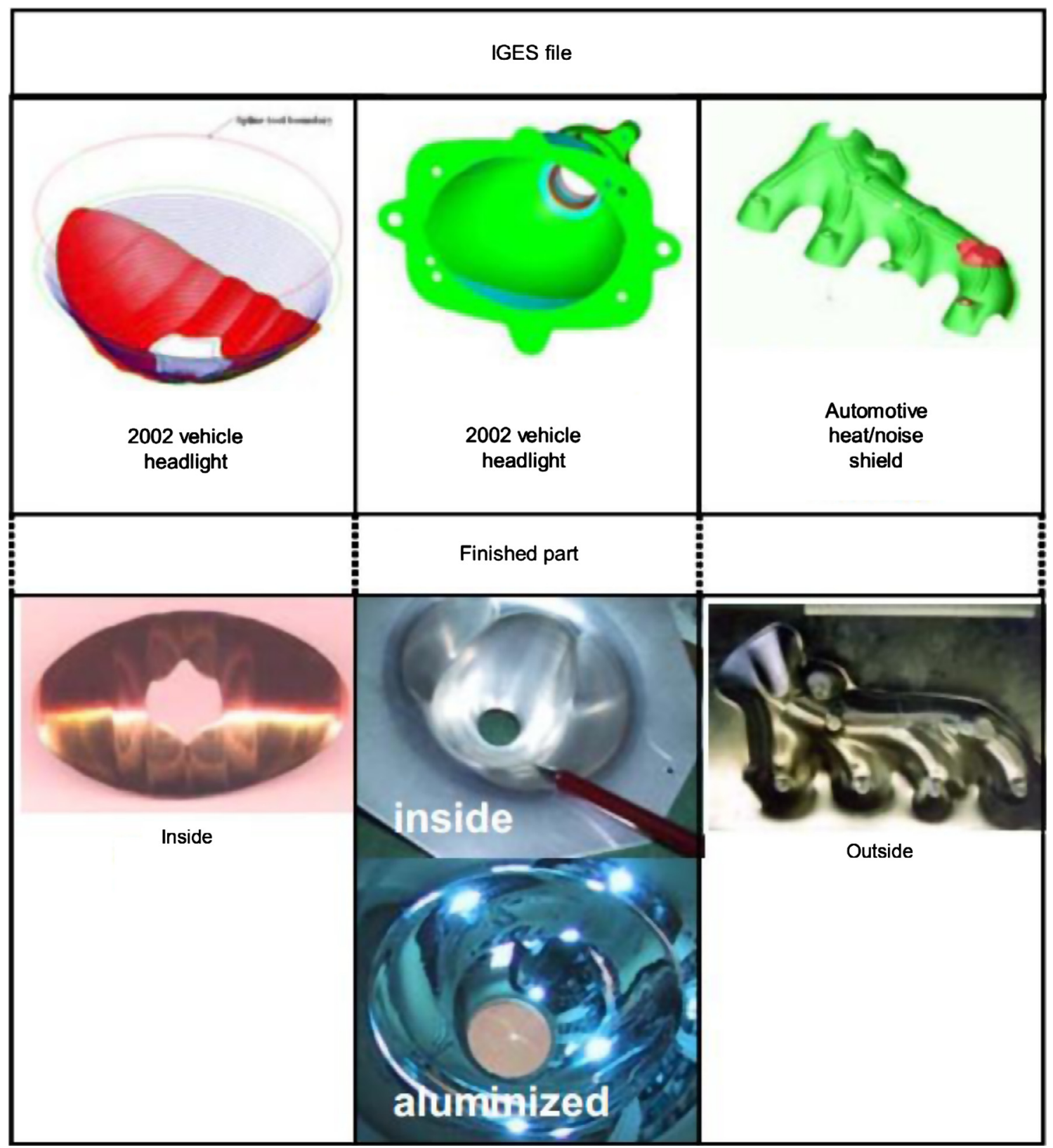

Figure 37. Rapid prototypes for automotive industries [61].

forming process. All the work was done in order to further enhance the formability and help to obtain the optimum processing parameters.

\subsection{Mechanism of the HF process}

It is widely believed that the main reason why the hydroforming process greatly improves the formability of the material is the introduction of through-thickness normal stress which causes superimposed hydrostatic pressure on the sheet metal.

Wu et al. [63] carried out a detailed analysis of uniaxial tension under superimposed hydrostatic pressure (see Figure 39) and demonstrated that the superimposed hydrostatic pressure lowers the true stress level at yielding by the amount of the superimposed hydrostatic pressure without affecting workhardening; the increase of sheet metal limit strains for any strain path under superimposed hydrostatic pressure has also been observed.

Fuchs [64] found that the forming limit of tube can be improved remarkably by applying liquid pressure to the inside and outside of the tube simultaneously. Cui et al. [65] designed a special experimental setup (see Figure 40) for double-sided tube hydroforming; the result shows that increase of external pressure has an effect on the fraction of grain boundaries, and on the number and size of the microvoids in the transition zone, and thus increases the critical effective strain in the transition zone. Liu et al. [66] simulated the effect of through-thickness normal stress on the sheet formability using the finite element software based on the ductile fracture criterion. 

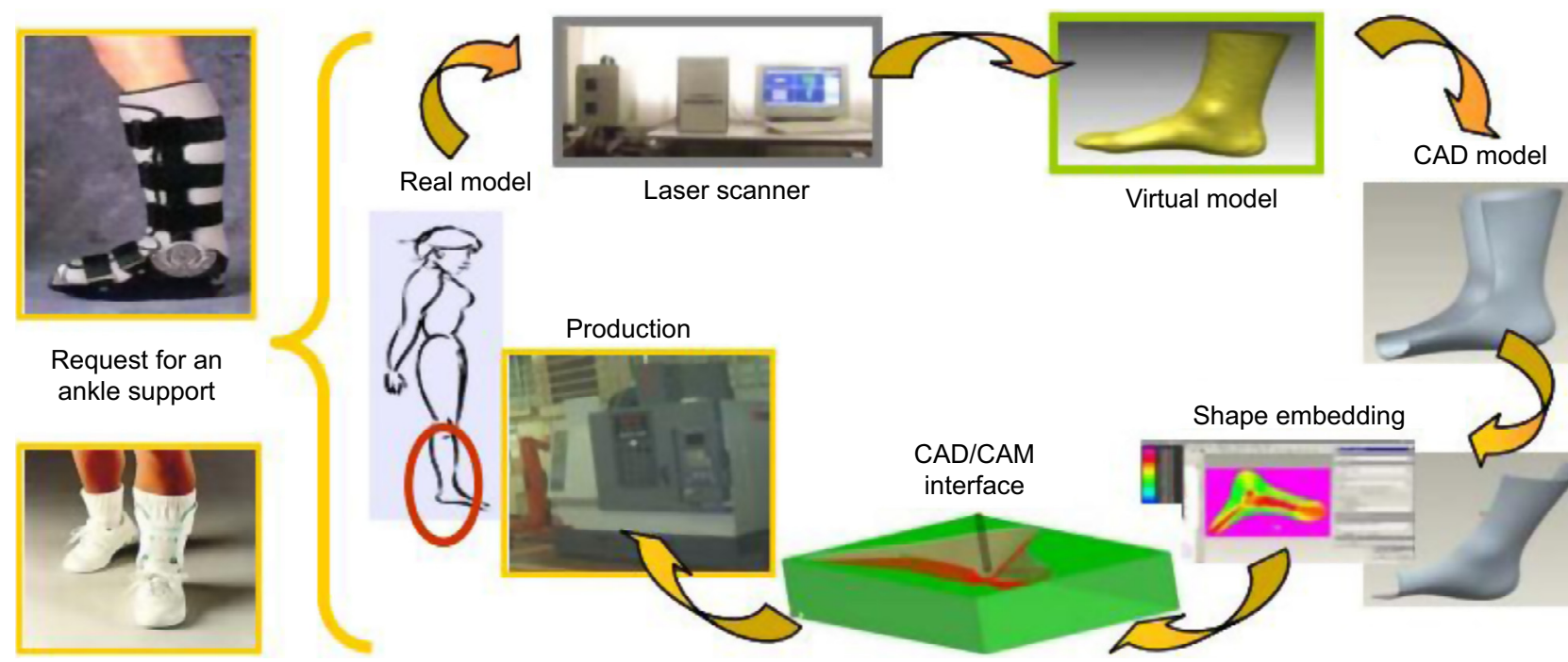

Figure 38. The manufacture of an ankle support [62].

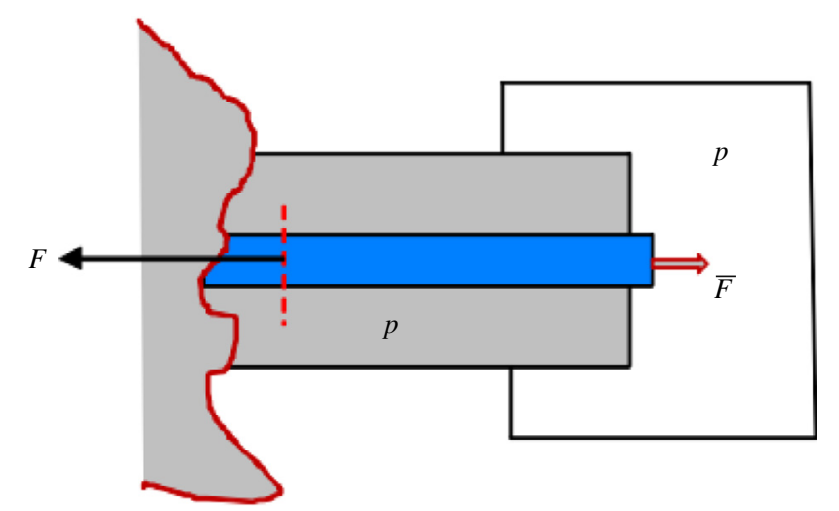

Figure 39. Schematic representation of a tensile sample in a pressure vessel [63].

Assempour et al. [67] studied the effect of normal stress on the prediction of the forming limit diagrams (FLDs) based on the Marciniak and Kuczynski (M-K) model with some modifications on the stress states for consideration of the normal stress effects; as expected, the compressive normal stress shifts the FLD upwards (see Figure 41). Michel and Picart [68] conducted tensile and hydraulic bulging tests (see Figure 42) to investigate the size effects on the constitutive behaviour of brass in sheet metal forming; both experiments showed the same trends, in that the flow curve decreases with a decrease of thickness: a new model considering the size effect was established.

\subsection{Mechanism of the SPF process}

The deformation mechanism of fine-grain aluminum alloys is generally accepted as grain-boundary-sliding (GBS) creep, which supplies a high strain-rate sensitivity for successful commercial superplastic forming operations [69, 70]. Aluminum alloy 5083 is the most common material in SPF operations and many researches have been focused on it.
Kulas et al. [70] studied the plastic deformation of seven 5083 commercial aluminum materials and found two mechanisms governing plastic deformation in AA5083: grainboundary-sliding (GBS) creep and solute-drag (SD) creep. Quantitative analysis of stress transients following different strain rates offers conclusive proof that SD creep dominates deformation at high strain rates and low temperature, whereas GBS creep occurs at high temperatures and low strain rates. Furthermore, deformation mechanism maps characterizing deformation in the fine-grained AA5083 materials were also constructed.

Kulas and Green [71] further investigated the failure mechanisms in AA5083 materials under uniaxial tension at elevated temperatures (see Figure 43) and discovered that the deformation dominated by grain-boundary-sliding creep leads to failure controlled by cavitation and the deformation dominated by solute-drag creep leads to failure controlled by flow localization. Further, the cavitation growth rate under SD creep is significantly less than that under GBS creep.

Li et al. [72] studied the superplastic behavior and microstructure of rolled Mg-Gd-Y-Zr alloy sheet and established that the high ductility of $\mathrm{Mg}-\mathrm{Gd}-\mathrm{Y}-\mathrm{Zr}$ alloy can be attributed to grain boundary sliding accommodated by dislocation motion assisted by lattice diffusion. Microstructural analysis indicated that the second phases exhibited the significant effect of pinning grain boundaries (see Figure 44).

\subsection{Mechanism of the AISF process}

The asymmetric single- and two-point incremental sheet forming processes are still in their infancy. Much research work remains to be done and to do this appropriate design of shapes (see Figure 36) need to be developed for the tests of FLDs, springback and roughness.

Jackson and Allwood [73] conducted special tests similar to those designed by Kalpakcioglu [74] using brazed plates in which a grid pattern was machined onto the cross-section of 


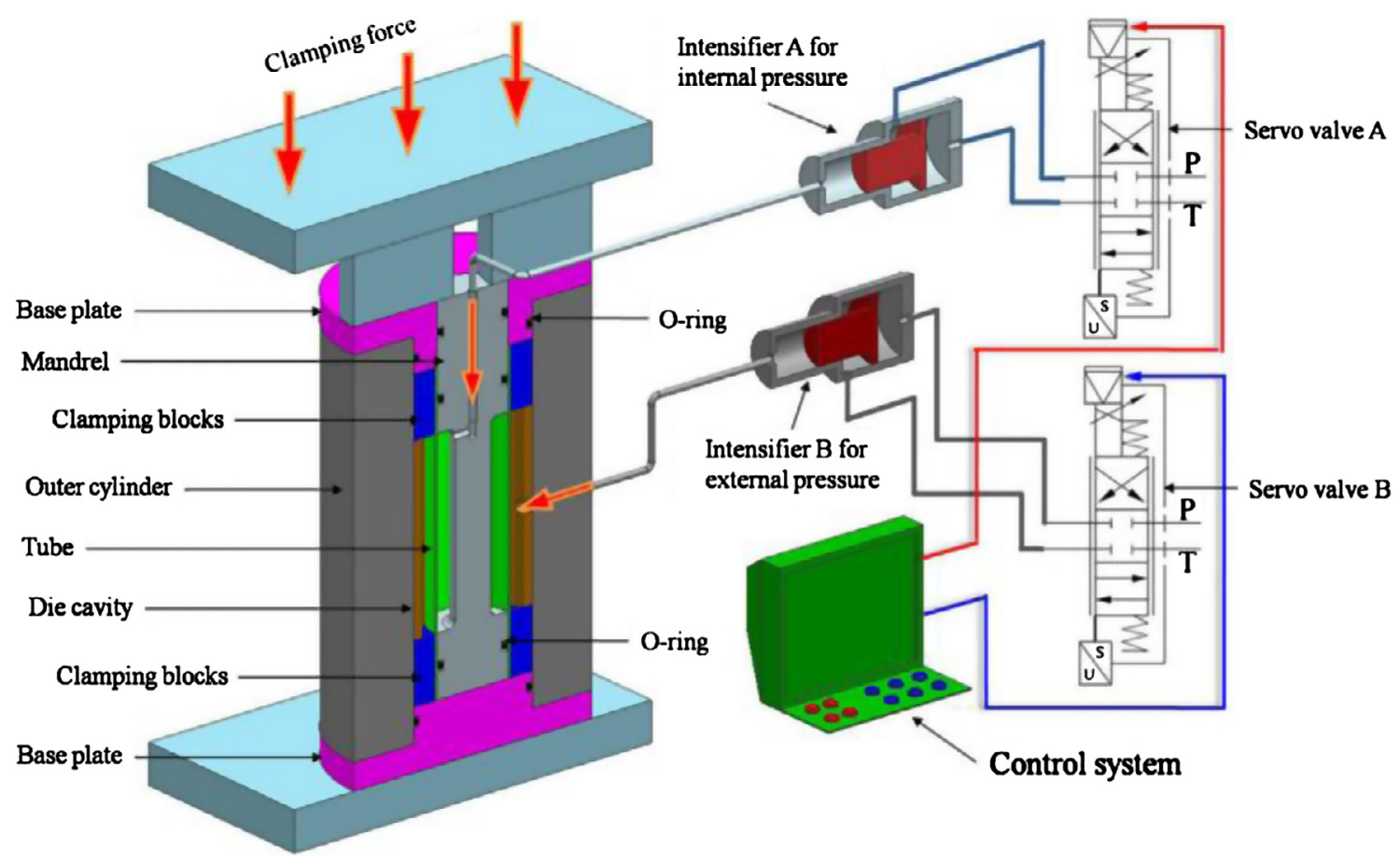

Figure 40. Experimental setup of double-sided tube hydroforming process [65].

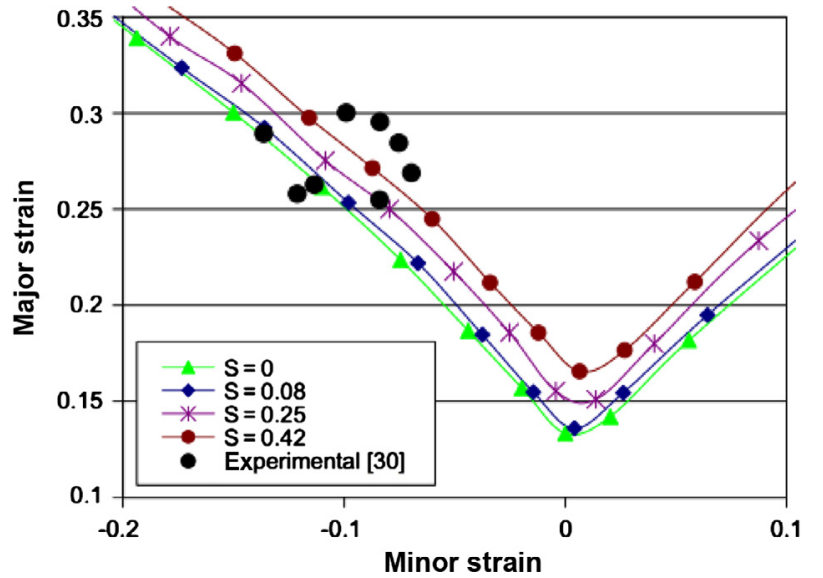

Figure 41. Effect of normal stress on shifting the FLDs for DP600 [67] ( $\mathrm{S}$ is normalized normal stress).

a half-plate (see Figure 45), which was then formed before being separated by heating. The deformation pattern of SPIF, TPIF and pressing was observed and it was revealed that the deformation mechanisms of both SPIF and TPIF are increasing stretching and shear in the radial-axial plane (perpendicular to the tool direction) and shear in the tool direction. Among these, shear in the tool direction, which is likely to be a result of friction between the tool and workpiece, is the most significant strain component. Silva et al. [75] studied the influence of process variables such as the tool radius to propose a new unified view on formability limits in the principal strain space and identified a critical threshold for the ratio between the thickness of the sheet and the radius of the tool that distinguishes between fracture with and without previous necking.

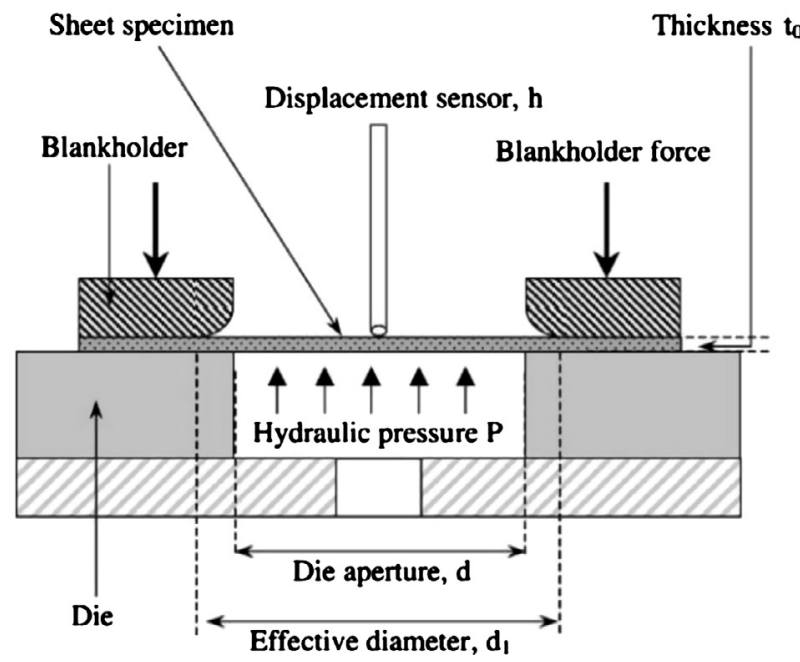

Figure 42. Hydraulic bulging machine [68].

Young and Jeswiet [76] used more than one shape to develop a non-traditional FLD for $1.21 \mathrm{~mm}$ thick AA 3003-0 formed by the AISF process. The non-traditional FLD could provide the designer with an additional method of judging if a design can be made in one pass with AISF. (The boundary for safe forming is shown by the dashed line in Figure 46.) Both Filice et al. [77] and Young [76] have found the same tendency of the boundary slope in the composite FLD.

Surface roughness is a major concern in a final product. In AISF the incremental step size, $\Delta z$, is the major factor in determining surface roughness $[78,79]$ which is shown in Figure 47. Junk et al. [79] also studied the effect of pitch $\Delta z$ and tool radius upon surface roughness and found the interrelationship between these parameters in the AISF process. 


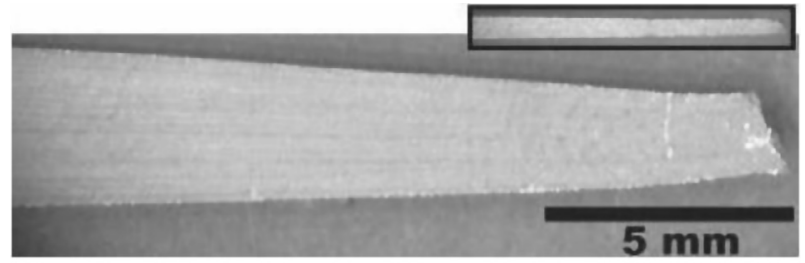

(a)

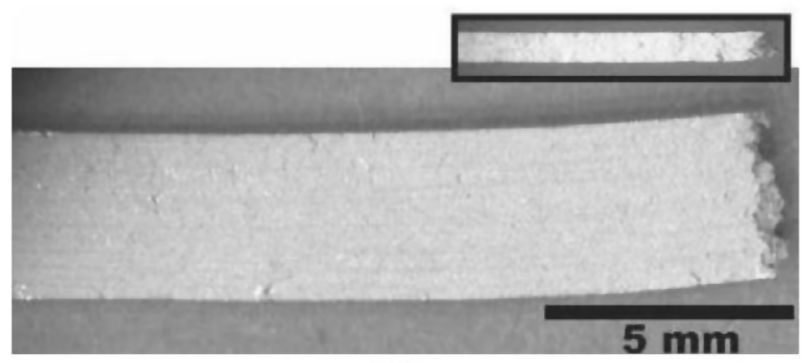

(b)

Figure 43. The failure regions of specimens are shown for (a) failure controlled by necking when deformation is by SD creep, (b) failure controlled by cavitation when deformation is by GBS creep [71].

(a)
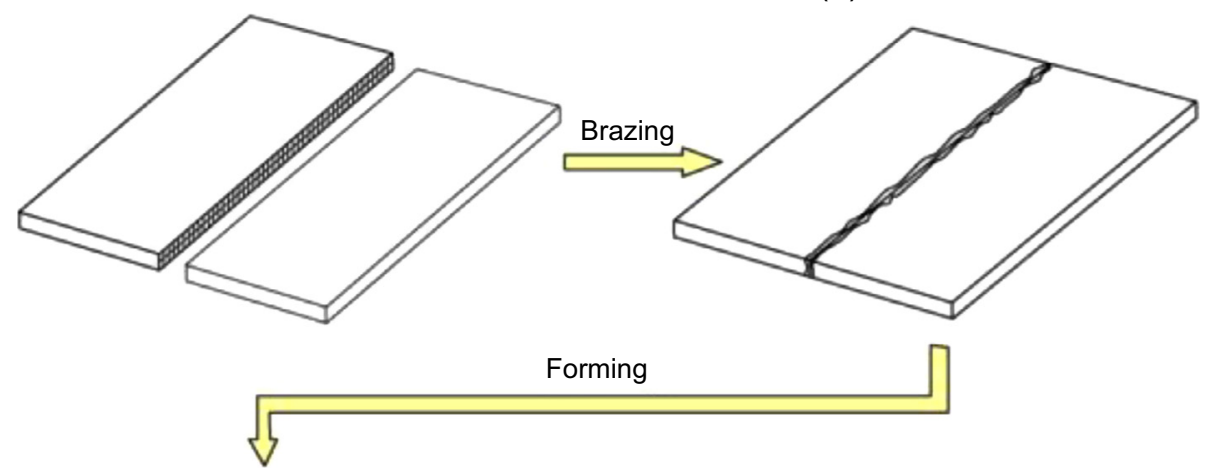

(c)
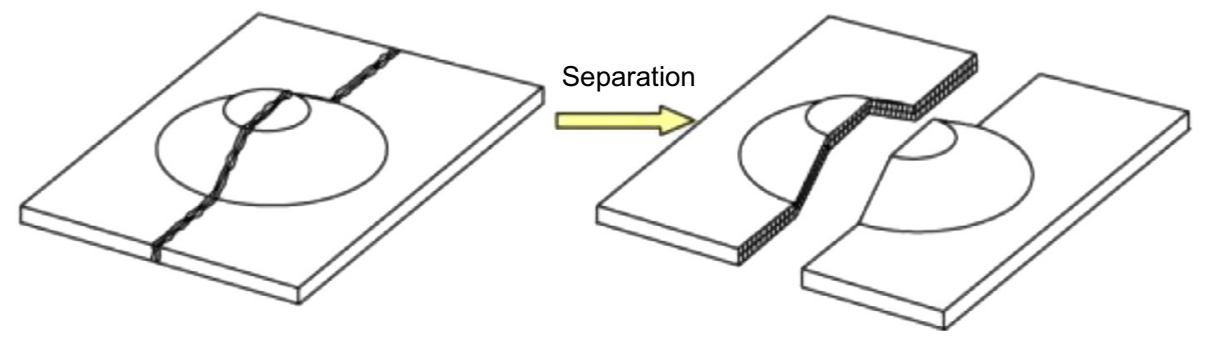

Figure 45. Schematic diagram of method for measuring through-thickness deformation by Jackson et al [73]. (a) Copper plate with gridded cross-section; (b) Plate with brazed joint; (c) Formed plate; (d) Separated plate with distorted grid pattern.

The springback effect is the main cause of the dimensional accuracy problems in the AISF process and has been widely investigated by several authors [80-83] Lasunon and Knight [80] developed a model to compare the accuracy of single-point incremental forming and double-point incremental forming and (d)

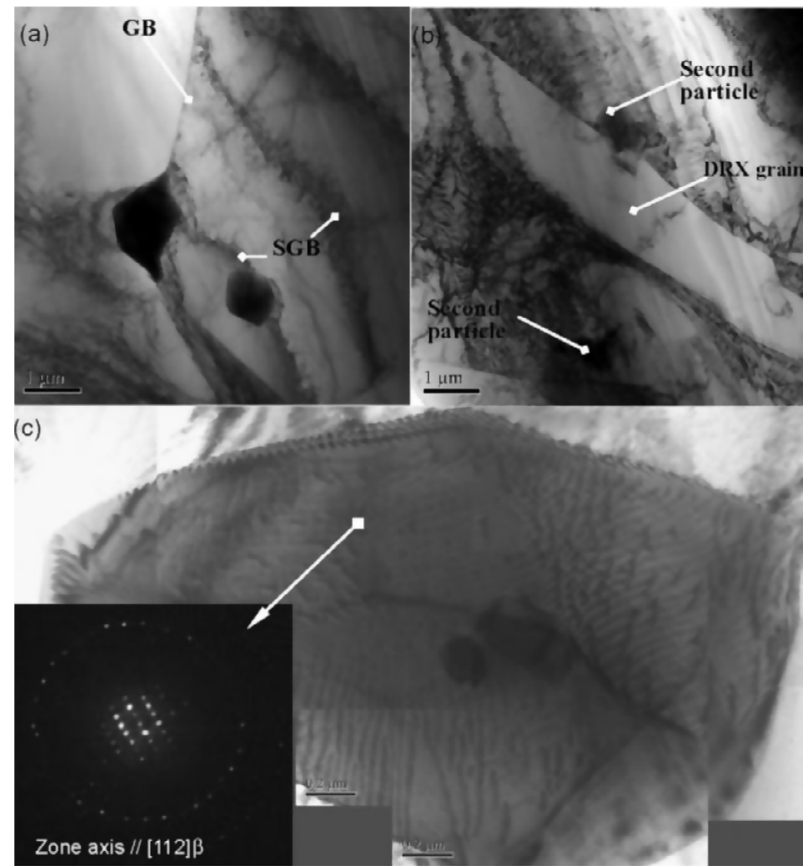

Figure 44. TEM images of the fractured specimen tested at $435{ }^{\circ} \mathrm{C}$ and $0.0005 \mathrm{~s}^{-1}$. (a) An example of grain boundary and subgrain boundary, (b) a strip recrystallized grain, (c) dislocation network within the $\beta$ phase [72].

(b)

found the FE model was in good agreement with experimental data (see Figure 48). Several strategies have been proposed to overcome accuracy problems such as systems with flexible programmable back support structures [82] and systems with a localized heating device [83]. 


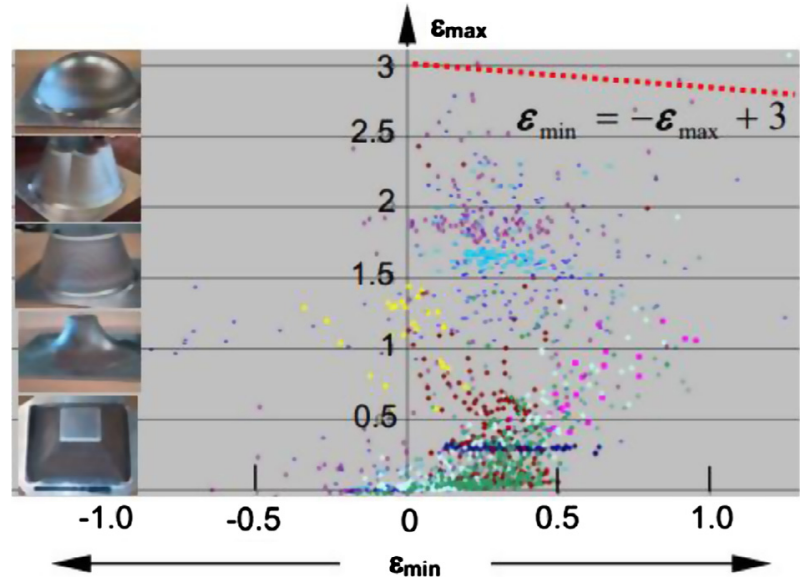

Figure 46. Non-traditional FLD formed by AISF method of $1.21 \mathrm{~mm}$ thick AA 3003-0 [76].

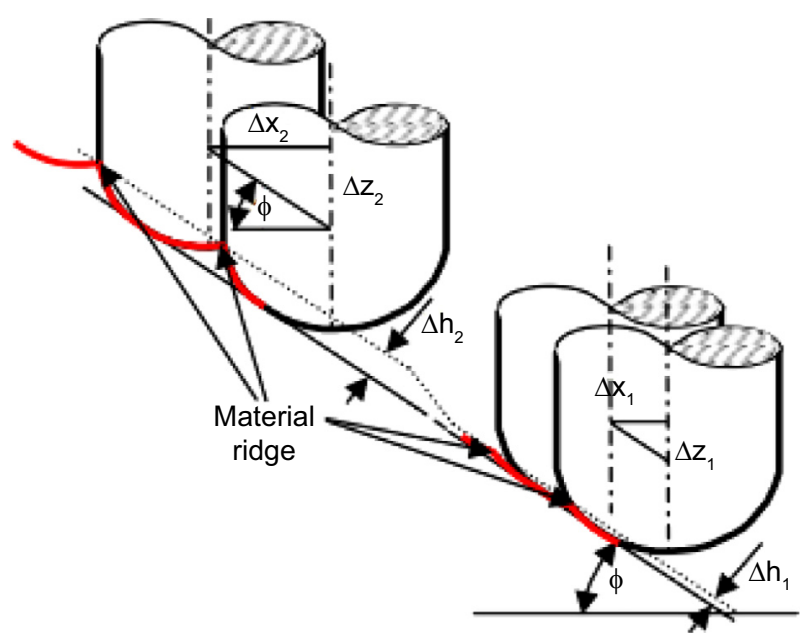

Figure 47. illustration of how the pitch size, $\Delta z$, influences roughness [60].

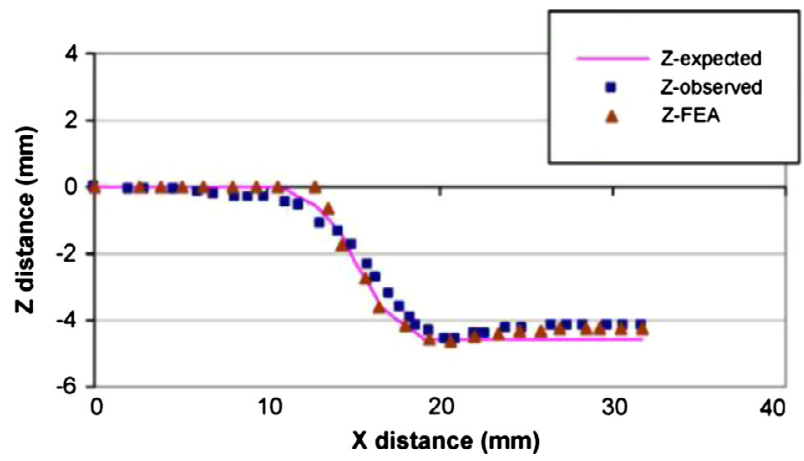

Figure 48. Comparison of profile plots gained from FEM and experimental results [80].

\subsection{The issues to be addressed}

A large amount of researches on these particular techniques has been undertaken to further enhance the formability of sheet and tube metal, although many problems still remains to be solved or improvements made to the aforementioned processes, among which some issues are listed below:

1. The accurate simulation of forming processes requires indepth comprehension of the friction mechanism between the blank and the tool, considering the effects of lubrication. With the application of lubrication, formability of metal sheet is increased as a consequence of reduced friction and it is possible to get more homogeneous thickness distributions [84].

2. Some of the special forming methods are conducted under relatively high temperatures, which may affect the microscopic and mechanical properties of the deformed components, so thermal influence should also be identified.

3. The FLD under complex strain paths is very important in forming sheet or tubular components with complex geometries has not yet been investigated. Also the influences of temperature and viscoplastic effect on FLD have attracted great interests from researchers recently [85].

4. Further research targeting onto improved hardware, tool path generation and control strategy development would be helpful in securing industrial opportunities.

\section{Conclusions}

Lightweight materials such as ultra-high-strength steel, titanium alloys and aluminum alloys are used widely to help to achieve the goal of emissions- and energy-reduction. In this review, recent developments on some particular sheet forming methods and their forming mechanisms (HF, VPF, SGMF, SPF/DB, IHF and AISF) are critically summarized with the objective of evaluating the different research results and indicating the current applications in the aerospace and automotive industries.

In general, some of these techniques have been used extensively to fabricate hard-to-form and complex-shaped components, i.e. hydroforming and superplastic forming processes, on the occasions where conventional forming methods cannot fulfill the requirements of the manufacturers. Some other techniques remain at the stage of laboratory research and need to be refined before spreading into modern industries.

\section{References}

1. J. Aspacher, Forming hardening concepts, 1st International Conference on Hot Sheet Metal Forming of High Performance Steel, Kassel, Germany, 2008, pp. 77-81.

2. R.R. Boyer, Applications of beta titanium alloys in airframes, Beta Titanium Alloys in the I990's, Warrendale, PA, TMS, 1993, pp. 335-346.

3. M. Peters, J. Kumpfert, C.H. Ward, C. Leyens, Titanium alloys for aerospace applications, Advanced Engineering Materials 5, 6 (2003) 419-427.

4. K. Martchek, Modelling more sustainable aluminium, The International Journal of Life Cycle Assessment 11, 1 (2006) 34-37. 
5. S. Toros, F. Ozturk, I. Kacar, Review of warm forming of aluminum-magnesium alloys, Journal of Materials Processing Technology 207, 1 (2008) 1-12.

6. T. Naka, G. Torikai, R. Hino, F. Yoshida, The effects of temperature and forming speed on the forming limit diagram for type 5083 aluminum-magnesium alloy sheet, Journal of Materials Processing Technology 113, 1 (2001) 648-653.

7. T. Naka, Y. Nakayama, T. Uemori, R. Hino, F. Yoshida, Effects of temperature on yield locus for 5083 aluminum alloy sheet, Journal of Materials Processing Technology 140, 1 (2003) 494-499.

8. D. Li, A. Ghosh, Tensile deformation behavior of aluminum alloys at warm forming temperatures, Materials Science and Engineering: A 352, 1 (2003) 279-286.

9. D. Li, A. Ghosh, Biaxial warm forming behavior of aluminum sheet alloys, Journal of Materials Processing Technology 145, 3 (2004) 281-293.

10. P. Cavaliere, Hot and warm forming of 2618 aluminium alloy, Journal of Light Metals 2, 4 (2002) 247-252.

11. R.R. Boyer, An overview on the use of titanium in the aerospace industry, Materials Science and Engineering: A 213, 1 (1996) 103-114.

12. C. Leyens, M. Peters, Titanium and titanium alloys, WileyVCH, Weinheim, 2003.

13. H. Karbasian, A.E. Tekkaya, A review on hot stamping, Journal of Materials Processing Technology 210, 15 (2010) 2103-2118.

14. D. Keith, HSS, AHSS and aluminum jockey for position in the race to cut auto curb weight, American Metal Market Monthly (2010) 1.

15. R. Kuziak, R. Kawalla, S. Waengler, Advanced high strength steels for automotive industry, Archives of Civil and Mechanical Engineering 8, 2 (2008) 103-117.

16. N.P. Lutsey, Review of technical literature and trends related to automobile mass-reduction technology, Institute of Transportation Studies, University of California, Davis, CA, 2010.

17. D. Anderson, New study finds increased use of advanced highstrength steels helps decrease overall vehicle weight, AISI News Release, Detroit, 2009.

18. M. Tolazzi, Hydroforming applications in automotive: a review, International Journal of Material Forming 3, 1 (2010) 307-310.

19. M. Koç, Hydroforming for advanced manufacturing, Woodhead Publishing, UK, 2008.

20. M. Koc, A. Taylan, An overall review of the tube hydroforming (THF) technology, Journal of Materials Processing Technology 108, 3 (2001) 384-393.

21. M. Ahmetoglu, K. Sutter, X.J. Li, T. Altan, Tube hydroforming: current research, applications and need for training, Journal of Materials Processing Technology 98, 2 (2000) 224-231.

22. B. Constantine, R. Roth, J.P. Clark, Substituting tubehydroformed parts for automotive stampings: an economic model, Journal of Modeling 53, 8 (2001) 33-38.

23. L.H. Lang, Z.R. Wang, D.C. Kang, S.J. Yuan, S.H. Zhang, J. Danckert, K.B. Nielsen, Hydroforming highlights: sheet hydroforming and tube hydroforming, Journal of Materials Processing Technology 151, 1 (2004) 165-177.

24. M. Kleiner, M. Geiger, A. Klaus, Manufacturing of lightweight components by metal forming, CIRP Annals-Manufacturing Technology 52, 2 (2003) 521-542.

25. P.J. Bolt, N.A.P.M. Lamboo, P.J.C.M. Rozier, Feasibility of warm drawing of aluminium products, Journal of Materials Processing Technology 115, 1 (2001) 118-121.
26. L.J. Rhoades, M.L. Rhoades, Die forming metallic sheet materials, US Patent No. 5,085,068, 4 Feb. 1992.

27. Z.J. Wang, J.G. Liu, X.Y. Wang, Z.Y. Hu, B. Guo, Viscous pressure forming (VPF): state-of-the-art and future trends, Journal of Materials Processing Technology 151, 1 (2004) 80-87.

28. Z.J. Wang, Z.R. Wang, H.F. Yang, Experimental investigation for viscous medium drawing of sheet metal under the condition of non-uniform blank holder force, Journal of Plasticity Engineering 6, 2 (1999) 50-52.

29. Z.R. Wang, X.Y. Wang, Z.J. Wang, Numerical simulation and experimental research on the forming of thin-walled complex parts with viscous pressure forming VPF, NUMISHEET, Korea, 2002, pp. 249-252.

30. M. Ahmetoglu, J. Hua, S. Kulukuru, T. Altan, Hydroforming of sheet metal using a viscous pressure medium, Journal of Materials Processing Technology 146, 1 (2004) 97-107.

31. L.B. Shulkin, R.A. Posteraro, M.A. Ahmetoglu, G.L. Kinzel, T. Altan, Blank holder force (BHF) control in viscous pressure forming (VPF) of sheet metal, Journal of Materials Processing Technology 98, 1 (2000) 7-16.

32. X.Y. Wang, J.C. Xia, G.A. Hu, Z.J. Wang, Z.R. Wang, Sheet bulging experiment with a viscous pressure-carrying medium, Journal of Materials Processing Technology 151, 1 (2004) 340-344.

33. J. Liu, Q. Peng, Y. Liu, Z. Wang, Viscous pressure bulging of aluminum alloy sheet at warm temperatures, Journal of Mechanical Science and Technology 21, 10 (2007) 1505-1511.

34. C.C. Zhao, G.J. Dong, H. Xiao, Y.S. Wang, New process of solid granule medium forming, Journal of Mechanical Engineering 8 (2009) 046.

35. G.J. Dong, C.C. Zhao, M.Y. Cao, Flexible-die forming process with solid granule medium on sheet metal, Transactions of Nonferrous Metals Society of China 23, 9 (2013) 2666-2677.

36. M.Y. Cao, C.C. Zhao, G.J. Dong, Numerical simulation on granules medium drawing process parameters of magnesium alloy sheet, The Chinese Journal of Nonferrous Metals 22, 11 (2012) 2992-2999.

37. M. Grüner, M. Merklein, Numerical simulation of hydro forming at elevated temperatures with granular material used as medium compared to the real part geometry, International Journal of Material Forming 3, 1 (2010) 279-282.

38. M. Grünerak, M. Merklein, Influences on the molding in hydroforming using granular material as a medium, AIP Conference Proceedings 1383 (2011) 645-652.

39. J. Bonet, A. Gil, R.D. Wood, R. Said, R.V. Curtis, Simulating superplastic forming, Computer Methods in Applied Mechanics and Engineering 195, 48 (2006) 6580-6603.

40. G.J. Davies, J.W. Edington, C.P. Cutler, K.A. Padmanabhan, Superplasticity: a review, Journal of Materials Science 5, 12 (1970) 1091-1102.

41. W.A. Backofen, I.R. Turner, D.H. Avery, Superplasticity in an Al-Zn alloy, Transactions of ASM 57, 4 (1964) 980-990.

42. J. Bonet, A. Gil, R.D. Wood, R. Said, R.V. Curtis, Simulating superplastic forming, Computer Methods in Applied Mechanics and Engineering 195, 48 (2006) 6580-6603.

43. A.J. Barnes, Superplastic forming 40 years and still growing, Journal of Materials Engineering and Performance 16, 4 (2007) 440-454.

44. L.D. Hefti, Commercial airplane applications of superplastically formed AA5083 aluminum sheet, Journal of Materials Engineering and Performance 16, 2 (2007) 136-141. 
45. L.D. Hefti, Using superplastic forming as a means of achieving cost benefits as well as enhancing aircraft performance, High Performance Metallic Materials for Cost Sensitive Applications, John Wiley and Sons, Hoboken, NJ, 2002 pp. 64-72.

46. R. Martin, D. Evans, Reducing costs in aircraft: the metals affordability initiative consortium, Journal of Modeling 52, 3 (2000) 24-28.

47. A.J. Barnes, Superplastic aluminum forming-expanding its techno-economic niche, Materials Science Forum 304 (1999) 785-796.

48. J. Wittenauer, T.G. Nieh, J. Wadsworth, A first report on superplastic gas-pressure forming of ceramic sheet, Scripta Metallurgica et Materialia 26, 4 (1992) 551-556.

49. T. Waniuk, J. Schroers, W.L. Johnson, Timescales of crystallization and viscous flow of the bulk glass-forming $\mathrm{Zr}$-Ti-Ni-Cu-Be alloys, Physical Review B 67, 18 (2003) 184203.

50. L.H. Lang, S.H. Wang, C.L. Yang, Investigation on the innovative impact hydroforming technology, The 11th International Conference on Numerical Methods in Industrial Forming Processes: NUMIFORM 2013 1532, 1 (2013) 791-798.

51. H. Tominga, M. Takamatsu, Hydropunch, a pneumatic-hydraulicforming machine, International Conference of the Center for High Energy Forming, Estes Park, USA, 1969.

52. B. Mason, Sheet metal forming for small batches, Bachelor thesis, University of Nottingham, May 1978.

53. W.C. Emmens, G. Sebastiani, A.H.V.D. Boogaard, The technology of incremental sheet forming - a brief review of the history, Journal of Materials Processing Technology 210, 8 (2010) 981-997.

54. B.T. Araghi, A. Göttmann, M. Bambach, G. Hirt, G. Bergweiler, J. Diettrich, M. Steiners, A. Saeed-Akbari, Review on the development of a hybrid incremental sheet forming system for small batch sizes and individualized production, Production Engineering 5, 4 (2011) 393-404.

55. M. Bambach, G. Hirt, S. Junk, Modelling and experimental evaluation of the incremental CNC sheet metal forming process, 7th International Conference on Computational Plasticity, Barcelona, Spain, 2003.

56. T.J. Kim, D.Y. Yang, Improvement of formability for the incremental sheet metal forming process, International Journal of Mechanical Sciences 42, 7 (2000) 1271-1286.

57. D. Leach, A.J. Green, A.N. Bramley, A new incremental sheet forming process for small batch and prototype parts, 9th International Conference on Sheet Metal, Leuven, 2001.

58. L. Filice, L. Fratini, F. Micari, Analysis of material formability in incremental forming, CIRP Annals-Manufacturing Technology 51, 1 (2002) 199-202.

59. S. Matsubara, Incremental backward bulge forming of a sheet metal with a hemispherical head tool-a study of a numerical control forming system II, Journal-Japan Society for Technology of Plasticity 35 (1994) 1311-1311.

60. J. Jeswiet, F. Micari, G. Hirt, A. Bramley, J. Duflou, J. Allwood, Asymmetric single point incremental forming of sheet metal, CIRP Annals-Manufacturing Technology 54, 2 (2005) 88-114.

61. J. Jeswiet, E. Hagan, Rapid prototyping of a headlight with sheet metal, Canadian Institute of Mining, Metallurgy and Petroleum, Canada, 2001, pp. 109-114.

62. F. Micari, Single point incremental forming: recent results, Seminar on Incremental Forming, Cambridge University, Cambridge, 22, 2004.

63. P.D. Wu, J.D. Embury, D.J. Lloyd, Y. Huang, K.W. Neale, Effects of superimposed hydrostatic pressure on sheet metal formability, International Journal of Plasticity 25, 9 (2009) 1711-1725.

64. F. Fuchs, Hydrostatic pressure - its role in metal forming, Mechanical Engineering 88, 4 (1966) 34-40.

65. X.L. Cui, X.S. Wang, S.J. Yuan, Deformation analysis of double-sided tube hydroforming in square-section die, Journal of Materials Processing Technology 214, 7 (2014) $1341-1351$.

66. J.G. Liu, Z.J. Wang, Q.Y. Meng, Numerical investigations on the influence of superimposed double-sided pressure on the formability of biaxially stretched AA6111-T4 sheet metal, Journal of Materials Engineering and Performance 21, 4 (2012) 429-436.

67. A. Assempour, H.K. Nejadkhaki, R. Hashemi, Forming limit diagrams with the existence of through-thickness normal stress, Computational Materials Science 48, 3 (2010) 504-508.

68. J.F. Michel, P. Picart, Size effects on the constitutive behaviour for brass in sheet metal forming, Journal of Materials Processing Technology 141, 3 (2003) 439-446.

69. T.G. Nieh, J. Wadsworth, O.D. Sherby, Superplasticity in metals and ceramics, Cambridge University Press, Cambridge, 2005.

70. M.A. Kulas, W.P. Green, E.M. Taleff, P.E. Krajewski, T.R. McNelley, Deformation mechanisms in superplastic AA5083 materials, Metallurgical and Materials Transactions A 36, 5 (2005) 1249-1261.

71. M.A. Kulas, W.P. Green, E.M. Taleff, P.E. Krajewski, T.R. McNelley, Failure mechanisms in superplastic AA5083 materials, Metallurgical and Materials Transactions A 37, 3 (2006) 645-655.

72. L. Li, X. Zhang, Y. Deng, C. Tang, Superplasticity and microstructure in Mg-Gd-Y-Zr rolled sheet, Journal of Alloys and Compounds 485, 1 (2009) 295-299.

73. K. Jackson, J. Allwood, The mechanics of incremental sheet forming, Journal of Materials Processing Technology 209, 3 (2009) 1158-1174.

74. S. Kalpakcioglu, On the mechanics of shear spinning, Journal of Engineering for Industry 83, 2 (1961) 125-130.

75. M.B. Silva, P.S. Nielsen, N. Bay, P.A.F. Martins, Failure mechanisms in single-point incremental forming of metals, The International Journal of Advanced Manufacturing Technology 56, 9-12 (2011) 893-903.

76. J. Jeswiet, D. Young, Forming limit diagrams for single-point incremental forming of aluminium sheet, Proceedings of the Institution of Mechanical Engineers, Part B: Journal of Engineering Manufacture 219, 4 (2005) 359-364.

77. L. Filice, L. Fratini, F. Micari, Analysis of material formability in incremental forming, CIRP Annals-Manufacturing Technology 51, 1 (2002) 199-202.

78. E. Hagan, J. Jeswiet, Analysis of surface roughness for parts formed by computer numerical controlled incremental forming, Proceedings of the Institution of Mechanical Engineers, Part B: Journal of Engineering Manufacture 218, 10 (2004) 1307-1312.

79. S. Junk, G. Hirt, I. Chouvalova, Forming strategies and tools in incremental sheet forming, Proceedings of the 10th International Conference on Sheet Metal, Belfast, 2003.

80. O. Lasunon, W.A. Knight, Comparative investigation of singlepoint and double-point incremental sheet metal forming processes, Proceedings of the Institution of Mechanical Engineers, Part B: Journal of Engineering Manufacture 221, 12 (2007) 1725-1732. 
81. S. Junk, G. Hirt, I. Chouvalova, Forming strategies and process modelling for CNC incremental sheet forming, CIRP AnnalsManufacturing Technology 53, 1 (2004) 203-206.

82. H. Meier, V. Smukala, O. Dewald, J. Zhang, Two point incremental forming with two moving forming tools, Key Engineering Materials 344 (2007) 599-605.

83. J.R. Duflou, B. Callebaut, J. Verbert, H.D. Baerdemaeker, Improved SPIF performance through dynamic local heating, International Journal of Machine Tools and Manufacture 48, 5 (2008) 543-549.
84. H.Y. Wu, C.H. Chiu, J.Y. Wang, S. Lee, Effect of lubrication on deformation characteristics of a superplastic 5083 Al alloy during bi-axial deformation, Materials Science and Engineering: A 427, 1 (2006) 268-273.

85. M. Serenelli, M. Bertinetti, P. Turner, J. Signorelli, A theoretical study on forming limit diagram predictions using viscoplastic polycrystalline plasticity models, Key Engineering Materials 473 (2011) 327-334.

Cite this article as: Lihui L, Kangning L, Cai G, Yang X, Guo C \& Bu G: A critical review on special forming processes and associated research for lightweight components based on sheet and tube materials. Manufacturing Rev. $2014,1,9$. 